\title{
Genetic Diversity of Transcriptional Activator-Like Effector Genes in Chinese Isolates of Xanthomonas oryzae pv. oryzicola
}

\author{
Zhi-Yuan Ji, Muhammad Zakria, Li-Fang Zou, Li Xiong, Zheng Li, Guang-Hai Ji, and Gong-You Chen
}

First, second, third, fourth, fifth, and seventh authors: School of Agriculture and Biology, Shanghai Jiao Tong University, Shanghai, 200240, China; and sixth author: Key Laboratory for Plant Pathology of Yunnan Province, College of Plant Protection, Yunnan Agricultural University, Kunming, 650201, China.

Accepted for publication 7 January 2014.

\begin{abstract}
Ji, Z.-Y., Zakria, M., Zou, L.-F., Xiong, L., Li, Z., Ji, G.-H., and Chen, G.-Y. 2014. Genetic diversity of transcriptional activator-like effector genes in Chinese isolates of Xanthomonas oryzae pv. oryzicola. Phytopathology 104:672-682.

Xanthomonas oryzae pv. oryzicola causes bacterial leaf streak (BLS), a devastating disease of rice in Asia countries. X. oryzae pv. oryzicola utilizes repertoires of transcriptional activator-like effectors (TALEs) to manipulate host resistance or susceptibility; thus, TALEs can determine the outcome of BLS. In this report, we studied genetic diversity in putative tale genes of $65 X$. oryzae pv. oryzicola strains that originated from nine provinces of southern China. Genomic DNAs from the 65 strains were digested with BamHI and hybridized with an internal fragment of avrXa3, a tale gene originating from the related pathogen, $X$. oryzae pv. oryzae, which causes bacterial leaf blight (BLB). Southern blot analysis indicated that the strains contained a variable number (9 to 22) of avrXa3-hybridizing fragments (e.g., putative tale genes). Based on the number and size of hybridizing bands, strains were classified into 14

genotypes (designated 1 to 14), and genotypes 3 and 10 represented 29.23 and $24.64 \%$ of the total, respectively. A high molecular weight BamHI fragment (HMWB; $\approx 6.0 \mathrm{~kb}$ ) was present in 12 of the 14 genotypes, and sequence analysis of the HMWB revealed the presence of a C-terminally truncated tale, an insertion element related to IS1403, and genes encoding phosphoglycerate mutase and endonuclease V. Primers were developed from the 6.0-kb HMWB fragment and showed potential in genotyping $X$. oryzae pv. oryzicola strains by polymerase chain reaction. Virulence of $X$. oryzae pv. oryzicola strains was assessed on 23 rice cultivars containing different resistance genes for BLB. The $X$. oryzae pv. oryzicola strains could be grouped into 14 pathotypes (I to XIV), and the grouping of strains was almost identical to the categories determined by genotypic analysis. In general, strains containing higher numbers of putative tale genes were more virulent on rice than strains containing fewer tales. The results also indicate that there are no gene-for-gene relationships between the tested rice lines and $X$. oryzae pv. oryzicola strains. To our knowledge, this is the first description of genetic diversity of $X$. oryzae pv. oryzicola strains based on tale gene analysis.
\end{abstract}

Bacterial leaf streak (BLS) caused by Xanthomonas oryzae pv. oryzicola is a major threat to rice production in Asia, Africa, and Australia $(12,13,28,30,33)$. The disease is especially serious in Asia, where hybrid rice is widely grown and causes yield losses up to 40 to $60 \%$ in severe epidemics $(16,17,30,52)$. The $X$. oryzae pv. oryzicola-rice interaction is a model system for studying how bacterial pathogens cause disease in rice $(30,52)$ and also provides important clues regarding the pathogenicity of $X$. oryzae pv. oryzae, which causes bacterial leaf blight (BLB) in rice. A classical gene-for-gene model for resistance exists for the $X$. oryzae pv. oryzae-rice pathosystem, and the most cost-effective and environmentally sound approach to combating BLB is the use of resistant rice varieties that interact with cognate avirulence (avr) genes in $X$. oryzae pv. oryzae $(2,14,19,28,39,43)$. However, with the exception of $R x o 1$, a resistance $(R)$ gene of maize origin that interacts with $X$. oryzae pv. oryzicola AvrRxol $(38,43,50)$, rice varieties with $R$ genes specific to $X$. oryzae pv. oryzicola are not available. Thus, studies to discover additional gene-for-gene interactions between $X$. oryzae pv. oryzicola and rice need to be undertaken.

Molecular fingerprinting techniques, including restriction fragment length polymorphism (RFLP); amplified fragment length

Corresponding author: G.-Y. Chen; E-mail address: gyouchen@sjtu.edu.cn

* The $e$-Xtra logo stands for "electronic extra" and indicates that the online version contains two supplemental figures.

http://dx.doi.org/10.1094/PHYTO-08-13-0232-R

(c) 2014 The American Phytopathological Society polymorphism (AFLP); and BOX-conserved repetive elements, enterobacterial repetitive intergenic consensus (ERIC), and repetitive sequence-based (Rep)-polymerase chain reaction (PCR), have been used to investigate genetic diversity in phytopathogenic bacteria, including $X$. oryzae pvs. oryzae and oryzicola $(1,13$, $18,22,34,35)$. However, polymorphisms identified using these approaches were not associated with bacterial virulence in host plants. In the last decade, comparative analysis of $X$. oryzae genomic sequences has revealed that many pathogenicity- and virulence-related genes are highly conserved between $X$. oryzae pvs. oryzae and oryzicola $(5,15,37)$. However, a notable exception is the variation in the number of transcriptional activator-like effector (tale) genes among the two pathovars $(15,40)$. For example, X. oryzae pv. oryzicola strains BLS256 and BLS303 contain $\approx 28$ and 33 tale genes (6), which exceed the numbers identified in $X$. oryzae pv. oryzae strains MAFF311018 (31), KACC10331 (23), and PXO99A (37). Previously, we tracked tale gene variations in different strains of $X$. oryzae pv. oryzae (44) and in Chinese isolates of $X$. citri subsp. citri, the citrus canker pathogen (48). It seems likely that the polymorphisms in tale genes may correlate with bacterial virulence in host plants. This prompts the following questions: do larger numbers of tale genes in $X$. oryzae pv. oryzicola strains result in a higher level of virulence? What is the distribution of tale genes in $X$. oryzae pv. oryzicola strains within rice-growing regions?

TALEs are a structurally and functionally distinct class of proteins present in Xanthomonas spp. that are secreted via the type III secretion system (T3SS) $(3,47)$. TALEs are delivered into plant nuclei and subsequently bind and activate upstream se- 
quences (UPT-box) present in $R$ or susceptibility $(S)$ genes $(3,21,41,45)$. TALEs share several common features, including an $\mathrm{N}$-terminal region required for secretion via the T3SS, a Cterminus containing nuclear localization signals (NLS), and an acidic activation domain (AD) typical of transcription factors. TALES generally contain a variable central domain of tandem, nearly identical 33- to 34-amino-acid (aa) repeats that confer DNA-binding specificity $(4,11,26,29)$. Residues at the 12 th and 13th positions are hypervariable and are known as repeat-variable diresidues (RVDs). The RVDs bind to specific, single nucleotides and, consequently, determine disease susceptibility or resistance. For example, PthXo1, a TALE in X. oryzae pv. oryzae strain PXO99 ${ }^{\mathrm{A}}$, binds to the UPT-box of the sucrose transporter gene OSSWEET11 (Os8N3). PthXo1 binding activates expression of OSSWEET11 and facilitates nutrient acquisition by the pathogen, thus resulting in susceptibility to BLB $(9,45)$. Similarly, AvrXa27 binds to the UPT-box of $\mathrm{Xa27}$ and activates its expression, thus leading to BLB resistance (14). However, similar interactions between TALEs and genes encoding resistance or susceptibility have not been identified for the $X$. oryzae pv. oryzicola-rice pathosystem. Thus, the survey of $X$. oryzae pv. oryzicola tale gene diversity conducted below will ultimately provide further insight into this host-pathogen interaction.

The objectives of this study were to evaluate the distribution of tale genes in $X$. oryzae pv. oryzicola strains collected in China and to investigate potential gene-for-gene interactions between $X$. oryzae pv. oryzicola and rice. This was undertaken by collecting $X$. oryzae pv. oryzicola isolates from nine rice-growing provinces, determining the number and size of tale genes by hybridization analysis, and assessing virulence in rice lines containing different $R$ genes for BLB.

\section{MATERIALS AND METHODS}

Isolation of bacterial strains. Rice leaves showing typical BLS symptoms in the field were collected and sent to us by local county technicians in local protection stations from major ricegrowing provinces of southern China from 2008 to 2009 (Table 1). One of five leaves in each sample was surface sterilized and crushed in $1.5-\mathrm{ml}$ microcentrifuge tubes containing $1 \mathrm{ml}$ of sterile distilled $\mathrm{H}_{2} \mathrm{O}\left(\mathrm{dH}_{2} \mathrm{O}\right)$ using a plastic pestle (Axygen, Hanzhou, China). Each bacterial suspension was serially diluted, plated to nutrient agar (NA) and NA containing 5\% skimmed milk (51) plates, and incubated at $28^{\circ} \mathrm{C}$ for 4 days. A single yellow, polysaccharide-rich bacterial representative of those showing extracellular protease haloes surrounding colonies isolated from each sample was selected, purified, and identified using $X$. oryzae pv. oryzicola-specific primers XoocF/XoocR (49) and hrpE3F/ hrpE3R (10) by colony PCR amplification. X. oryzae pv. oryzicola isolates were individually infiltrated into tobacco leaves (Nicotiana benthamiana) for hypersensitive response (HR) detection using needleless syringes and inoculated into rice line IR24 (susceptible to X. oryzae pv. oryzicola) using the leafneedling method (52). Strains causing typical HR in tobacco and BLS symptoms in rice IR24 were maintained at $-80^{\circ} \mathrm{C}$ in nutrient broth containing $30 \%$ glycerol for further investigation. In total, $65 X$. oryzae pv. oryzicola strains were collected from different rice samples for which detailed genetic backgrounds were known by the local technicians.

DNA extraction and Southern blot analysis. Total genomic DNAs from $65 X$. oryzae pv. oryzicola strains were extracted using the AxyPrep Bacterial Genomic DNA Miniprep Kit (Axygen). DNA samples $(3 \mu \mathrm{g})$ were digested with BamHI at $37^{\circ} \mathrm{C}$ for $4 \mathrm{~h}$, separated in $1.2 \%$ agarose gels, and then transferred to Hybond $\mathrm{N}^{+}$nylon membranes (Millipore, Billerica, MA). The probe was a digoxigenin-labeled 1,368-bp internal SphI fragment of avrXa3 (GenBank accession number AY129298.1) originating from $X$. oryzae pv. oryzae (44). This 1,368-bp fragment contains
8.5 tandem repeats of a 102-bp sequence and part of the leucine zipper (LZ) coding region (44). This internal fragment of avrXa3 was used as a probe to detect the number and size of tale genes in each $X$. oryzae pv. oryzicola strain. DNA labeling, hybridization, and detection were performed as recommended by the manufacturer (Roche, Sweden); all hybridization experiments were repeated twice.

Analysis of virulence. Because $X$. oryzae pv. oryzicola and $X$. oryzae pv. oryzae are close pathovars in rice (30), we assumed that $R$ genes for BLB in different rice lines against $X$. oryzae pv. oryzae containing corresponding tale genes may occasionally match a tale gene contained in these $65 X$. oryzae pv. oryzicola strains. To investigate whether there are rice lines resistant to $X$. oryzae pv. oryzicola infection, 23 rice lines containing different $R$ genes for BLB (41) were used for virulence assessment of the collected $X$. oryzae pv. oryzicola strains (Table 1). Rice plants were maintained in a greenhouse at Shanghai Jiao Tong University with a photoperiod of $14 \mathrm{~h}$ of light $\left(30^{\circ} \mathrm{C}\right)$ and $10 \mathrm{~h}$ of darkness $\left(25^{\circ} \mathrm{C}\right)$, while tobacco plants were grown in the greenhouse with a photoperiod of $14 \mathrm{~h}$ of light $\left(24^{\circ} \mathrm{C}\right)$ and $10 \mathrm{~h}$ of darkness $\left(18^{\circ} \mathrm{C}\right) . X$. oryzae pv. oryzicola strains were grown overnight on NA plates at $28^{\circ} \mathrm{C}$, washed twice, and resuspended in $\mathrm{dH}_{2} \mathrm{O}$ to an optical density at $600 \mathrm{~nm}=0.6$ prior to inoculation. Leaves of 4-week-old rice seedlings were infiltrated with bacterial suspensions using a needleless syringe (51), and each leaf was infiltrated with the same strain in three different spots. Five secondary leaves were inoculated with each strain. Lesion lengths were measured 6 days after inoculation. Virulence levels were evaluated according to lesion size as follows: level 0 , lesions $\leq 0.1 \mathrm{~cm}$; level 1 , lesions 0.1 to $0.5 \mathrm{~cm}$; level $2,0.5$ to $1.5 \mathrm{~cm}$; level $3,1.5$ to $2.0 \mathrm{~cm}$; level $4,2.0$ to $2.5 \mathrm{~cm}$; level $5,2.5$ to $3.0 \mathrm{~cm}$; level $6,3.0$ to $3.5 \mathrm{~cm}$; level $7,3.5$ to $4.0 \mathrm{~cm}$; level $8,4.0$ to $4.5 \mathrm{~cm}$; level $9,4.5$ to $5.0 \mathrm{~cm}$; and level 10 , lesions $>5.0 \mathrm{~cm}$ in length. Based on the virulence assessment, unweighted pair-group arithmetic method (UPGMA) cluster analysis (18) of simple matching coefficients (a similarity value that can be applied to measure qualitative, multistate parameters) was used to quantify $X$. oryzae pv. oryzicola virulence in different rice lines. All virulence assays were performed in three independent experiments.

Data analysis. The number and size of tale genes were converted to binary form, where 1 indicates the presence of a band and 0 indicates the absence of the corresponding band. An intensely hybridizing band was considered to contain more than one tale gene in the analysis. Bands separated by $>2 \mathrm{~mm}$ were considered different. Genetic diversity was measured by the percentage of variable bands and the Shannon diversity index (25). Jaccard similarity coefficients for pairs of $X$. oryzae pv. oryzicola strains were calculated using NTSYS (v. 2.1; Exeter Software, Setauket, NY) using SIMQUAL with the Dice coefficient; results were subjected to cluster analysis using the UPGMA (18)

The diversity of virulent pathotypes was measured by a simple matching coefficient similarity matrix, based on a matrix of virulence levels generated by the SIMQUAL routine in NTSYS (18). Cluster analysis was performed using UPGMA with the arithmetic mean method in the SAHN routine of NTSYS (18). To estimate the strength of the groups generated by cluster analysis, bootstrap analysis was performed on 1.000 replications using Winboot software (http://books.irri.org/DPS14_content.pdf).

Isolation and sequencing of large tale-containing Bam $\mathrm{HI}$ fragments. The fine structure of putative tale-containing $6.0-\mathrm{kb}$ high molecular weight bands (HMWBs) was investigated in $X$. oryzae pv. oryzicola strains RS105 and JSB2-24. Genomic DNA from these two strains was digested with BamHI, and 6.0to $8.0-\mathrm{kb}$ fragments were purified from $1.0 \%$ agarose gels. The purified HMWBs were then ligated into BamHI-digested vector pBluescript II SK(-) for sequencing (Invitrogen, Shanghai, China). The full-length HMWB sequences from RS105 and 
TABLE 1. Lesion lengths of the tested Xanthomonas oryzae pv. oryzicola strains in 23 different rice varieties

\begin{tabular}{|c|c|c|c|c|c|c|c|c|c|c|c|c|}
\hline \multirow[b]{3}{*}{ Strains ${ }^{\mathrm{a}}$} & \multicolumn{12}{|c|}{ Lesion length $(\mathrm{cm})$ for rice lines and genes shown ${ }^{\mathrm{b}}$} \\
\hline & Asominor & BJ1 & Cas209 & DV85 & IR24 & IR26 & IRBB1 & IRBB2 & IRBB3 & IRBB4 & IRBB5 & IRBB7 \\
\hline & Xa17 & $x a 5, x a 13$ & Xa10 & $X a 7$ & Xa18 & Xa4 & Xa1 & $X a 2$ & Xa3 & Xa4 & $x a 5$ & $X a 7$ \\
\hline SCB04-1 & 1.44 & 0.92 & 1.48 & 0.42 & 1.04 & 1.76 & 0.9 & 1.84 & 0.78 & 0.78 & 1.34 & 0.86 \\
\hline YNB1-1 & 1.4 & 0.74 & 1.94 & 0.34 & 1.42 & 1.28 & 0.38 & 2.34 & 0.48 & 2.18 & 1.32 & 2.18 \\
\hline YNB9-3 & 1.38 & 1.66 & 2.74 & 0.82 & 2.98 & 2.38 & 2.84 & 2.3 & 1.82 & 1.36 & 1.78 & 2.2 \\
\hline SCB9-02 & 1.48 & 1.78 & 2.86 & 0.68 & 2.56 & 2.14 & 2.86 & 2.26 & 1.34 & 1.34 & 1.58 & 2.28 \\
\hline ZJB9-2 & 1.24 & 1.94 & 2.88 & 0.64 & 2.64 & 2.32 & 2.82 & 2.36 & 1.28 & 1.34 & 2.2 & 2.28 \\
\hline HANB9-1 & 1.38 & 1.96 & 2.48 & 0.84 & 2.86 & 2.44 & 2.66 & 2.2 & 1.72 & 1.38 & 1.8 & 1.74 \\
\hline JSB2-24 & 1.3 & 1.82 & 2.92 & 0.82 & 2.52 & 1.9 & 2.98 & 2.08 & 1.34 & 1.44 & 1.8 & 2.1 \\
\hline GDB3-143 & 1.48 & 1.42 & 2.74 & 0.8 & 2.5 & 2.44 & 2.52 & 2.36 & 1.46 & 1.26 & 2.26 & 2.28 \\
\hline HNB16-97 & 1.54 & 1.64 & 2.4 & 0.76 & 2.94 & 2.24 & 2.86 & 2.7 & 1.36 & 1.3 & 2.24 & 2.32 \\
\hline HNB16-100 & 1.48 & 1.78 & 2.9 & 0.58 & 2.76 & 2.5 & 2.68 & 2.18 & 1.3 & 1.32 & 1.78 & 1.72 \\
\hline AHB4-204 & 1.36 & 1.6 & 2.54 & 0.68 & 2.66 & 2.48 & 2.42 & 2.24 & 1.32 & 1.8 & 1.8 & 1.82 \\
\hline HNB16-46 & 1.48 & 1.88 & 2.56 & 0.66 & 2.68 & 2.16 & 2.74 & 2.76 & 1.34 & 1.46 & 2.24 & 2.2 \\
\hline AHB4-147 & 1.64 & 1.62 & 2.54 & 0.64 & 2.6 & 2.28 & 2.82 & 2.2 & 1.44 & 1.66 & 1.74 & 1.78 \\
\hline AHB4-205 & 1.12 & 1.34 & 2.64 & 0.54 & 2.78 & 2.66 & 2.08 & 2.4 & 1.84 & 1.4 & 2.18 & 2.22 \\
\hline AHB4-118 & 1.28 & 1.44 & 2.84 & 0.76 & 2.8 & 2.88 & 2.42 & 2.22 & 1.56 & 1.78 & 2.38 & 1.8 \\
\hline AHB3-7 & 1.38 & 1.82 & 2.9 & 0.54 & 2.76 & 2.48 & 2.64 & 2.14 & 1.24 & 1.56 & 1.86 & 1.8 \\
\hline AHB2-131 & 1.54 & 2.26 & 2.64 & 1.28 & 2.72 & 2.74 & 2.36 & 2.36 & 1.8 & 1.8 & 2.28 & 2.16 \\
\hline GDB8-47 & 1.58 & 1.52 & 2.58 & 0.94 & 2.98 & 2.72 & 2.06 & 2.78 & 1.34 & 1.78 & 2.42 & 2.32 \\
\hline ZJB01-25 & 1.38 & 1.36 & 2.96 & 1.32 & 2.5 & 2.68 & 2.24 & 2.2 & 1.3 & 1.78 & 1.82 & 1.8 \\
\hline HNB12-26 & 1.58 & 1.62 & 2.78 & 0.84 & 2.68 & 2.12 & 2.38 & 2.28 & 1.78 & 1.54 & 1.76 & 1.82 \\
\hline SCB9-5 & 1.42 & 1.74 & 2.68 & 0.68 & 2.66 & 2.42 & 2.48 & 2.24 & 1.4 & 1.62 & 1.72 & 2.22 \\
\hline HNB3-17 & 3.26 & 1.42 & 2.6 & 2.62 & 3.7 & 1.5 & 1.44 & 1.92 & 2.36 & 0.8 & 1.84 & 1.24 \\
\hline YNB01-3 & 3.42 & 1.44 & 2.9 & 2.68 & 2.16 & 1.64 & 1.66 & 1.62 & 1.8 & 0.64 & 1.82 & 1.78 \\
\hline JSB5-2 & 4.66 & 1.36 & 2.06 & 1.48 & 2.32 & 3.52 & 1.88 & 3.14 & 1.34 & 2.2 & 3.54 & 2.74 \\
\hline SCB04-2 & 1.24 & 0.6 & 1.42 & 1.76 & 3.02 & 3.12 & 3.36 & 2.24 & 1.74 & 2.4 & 2.7 & 1.78 \\
\hline YNB63 & 1.4 & 0.82 & 1.46 & 1.82 & 3.28 & 3.84 & 3.4 & 2.3 & 1.84 & 2.4 & 2.76 & 1.64 \\
\hline GXB9-31 & 1.42 & 0.72 & 1.38 & 1.68 & 3.48 & 3.12 & 3.04 & 1.92 & 1.66 & 2.34 & 2.7 & 1.84 \\
\hline SCB9-24 & 1.44 & 0.7 & 1.64 & 1.7 & 3.38 & 3.22 & 3.46 & 2.16 & 2.24 & 2.16 & 2.78 & 1.84 \\
\hline HANB9-23 & 1.58 & 0.66 & 1.46 & 1.76 & 3.1 & 3.06 & 3.74 & 2.24 & 1.78 & 1.82 & 2.28 & 1.66 \\
\hline SCB9-18 & 1.46 & 0.56 & 1.48 & 1.88 & 3.5 & 3.5 & 3.82 & 2.4 & 2.36 & 2.18 & 2.78 & 1.66 \\
\hline HANB8-2 & 1.54 & 0.64 & 1.48 & 1.74 & 2.58 & 3.24 & 3.96 & 2.16 & 1.72 & 2.44 & 2.26 & 1.72 \\
\hline RS85 & 1.2 & 0.86 & 1.68 & 1.52 & 3.24 & 3.38 & 3.84 & 2.2 & 2.14 & 1.7 & 2.84 & 1.86 \\
\hline ZJB9-46 & 2.46 & 0.74 & 1.74 & 1.9 & 2.72 & 3.38 & 2.62 & 3.56 & 3.54 & 1.8 & 2.76 & 2.22 \\
\hline YNB9-41 & 2.38 & 0.66 & 1.72 & 1.66 & 2.82 & 3.48 & 2.92 & 3.16 & 2.74 & 1.96 & 2.56 & 2.28 \\
\hline SCB9-39 & 2.34 & 0.8 & 1.72 & 1.6 & 2.62 & 3.42 & 3.02 & 3.64 & 3.64 & 1.76 & 2.7 & 2.3 \\
\hline YNB21 & 1.34 & 0.58 & 2.4 & 1.76 & 2.42 & 2.08 & 2.68 & 1.32 & 1.72 & 1.74 & 1.36 & 2.28 \\
\hline GDB3-12 & 1.46 & 0.62 & 2.42 & 1.62 & 2.4 & 2.16 & 2.86 & 1.22 & 1.62 & 1.56 & 1.48 & 2.42 \\
\hline GXB3-4 & 1.36 & 0.72 & 2.34 & 1.82 & 2.04 & 2.64 & 2.82 & 1.42 & 1.7 & 1.68 & 1.34 & 2.4 \\
\hline GXB3-23 & 1.38 & 0.8 & 2.46 & 1.48 & 2.44 & 2.8 & 2.7 & 1.3 & 2.18 & 1.82 & 1.76 & 2.2 \\
\hline GXB3-8 & 1.3 & 0.82 & 2.84 & 1.82 & 2.14 & 2.32 & 2.6 & 1.28 & 2.28 & 2.12 & 1.38 & 2.34 \\
\hline GDB3-092 & 1.42 & 0.8 & 2.48 & 1.84 & 2.08 & 2.86 & 2.74 & 1.8 & 2.38 & 2.24 & 1.8 & 2.48 \\
\hline GDB3-087 & 1.44 & 0.86 & 2.36 & 1.44 & 2.26 & 2.3 & 3.16 & 1.3 & 1.78 & 2.26 & 1.38 & 2.3 \\
\hline JSB3-120 & 1.26 & 0.96 & 2.9 & 1.14 & 2.12 & 2.02 & 2.62 & 1.3 & 2.32 & 1.78 & 1.36 & 2.4 \\
\hline JSB3-102 & 1.28 & 1.26 & 2.28 & 1.24 & 2.46 & 2.5 & 2.78 & 1.74 & 2.46 & 2.22 & 1.78 & 2.3 \\
\hline YNB9-53 & 1.22 & 0.78 & 2.4 & 1.44 & 2.08 & 2.24 & 3.28 & 1.26 & 2.28 & 1.68 & 1.38 & 2.22 \\
\hline HANB9-48 & 1.48 & 0.58 & 2.36 & 1.36 & 2.1 & 2.36 & 2.84 & 1.38 & 1.74 & 1.84 & 1.42 & 2.26 \\
\hline HANB9-35 & 1.28 & 0.64 & 2.28 & 1.26 & 2.38 & 2.42 & 3.12 & 1.92 & 1.82 & 1.66 & 1.74 & 2.36 \\
\hline GXB9-32 & 1.36 & 0.86 & 2.26 & 1.22 & 2.42 & 2.24 & 2.74 & 1.28 & 1.54 & 1.64 & 1.32 & 2.18 \\
\hline GXB1-6 & 1.28 & 0.76 & 2.76 & 1.34 & 2.24 & 2.18 & 2.66 & 1.78 & 1.64 & 1.8 & 1.42 & 1.72 \\
\hline JSB3-28 & 1.68 & 0.98 & 2.36 & 1.3 & 2.32 & 2.22 & 2.86 & 1.32 & 1.56 & 1.68 & 1.38 & 2.24 \\
\hline AHB1-58 & 1.26 & 0.7 & 2.52 & 1.44 & 2.16 & 2.44 & 3.02 & 1.32 & 1.66 & 1.88 & 1.74 & 2.3 \\
\hline RS105 & 2.42 & 1.52 & 2.58 & 2.56 & 2.66 & 3.08 & 2.94 & 1.28 & 1.44 & 0.68 & 0.84 & 1.8 \\
\hline ZJB01-19 & 1.84 & 1.44 & 1.84 & 1.52 & 2.16 & 4.46 & 3.44 & 3.68 & 1.72 & 1.8 & 1.32 & 1.42 \\
\hline ZJB01-12 & 1.78 & 1.32 & 1.88 & 1.68 & 2.12 & 4.12 & 3.08 & 3.7 & 1.64 & 1.44 & 1.34 & 1.32 \\
\hline YNB9-44 & 1.92 & 1.44 & 1.94 & 1.66 & 2.46 & 4.36 & 3.16 & 3.54 & 1.72 & 1.68 & 1.18 & 1.24 \\
\hline ZJB9-38 & 1.98 & 1.32 & 1.94 & 1.18 & 2.36 & 4.34 & 3.22 & 3.76 & 1.48 & 1.84 & 1.86 & 1.46 \\
\hline SCB9-25 & 1.74 & 1.48 & 1.88 & 1.82 & 2.26 & 4.24 & 2.84 & 3.3 & 1.78 & 1.78 & 1.38 & 1.3 \\
\hline RS119 & 2.86 & 1.66 & 2.8 & 2.9 & 2.78 & 2.38 & 2.92 & 2.36 & 2.3 & 1.78 & 1.7 & 1.8 \\
\hline HANB10-10 & 2.96 & 2.06 & 2.8 & 2.76 & 2.6 & 2.24 & 3.3 & 2.24 & 1.8 & 1.32 & 1.7 & 1.52 \\
\hline HNB1-19 & 2.68 & 2.12 & 2.94 & 2.4 & 2.56 & 2.24 & 2.88 & 2.38 & 2.18 & 1.78 & 2.26 & 1.78 \\
\hline HANB01-12 & 2.9 & 2.16 & 2.84 & 1.68 & 2.84 & 2.36 & 2.46 & 2.24 & 2.28 & 1.36 & 2.36 & 1.66 \\
\hline HNB07-3 & 3.96 & 2.26 & 1.46 & 2.16 & 2.56 & 2.38 & 2.42 & 2.82 & 1.38 & 2.36 & 1.46 & 1.66 \\
\hline GDB3-4 & 1.26 & 0.96 & 1.76 & 1.26 & 1.74 & 3.1 & 1.82 & 2.78 & 2.2 & 2.28 & 3.14 & 1.26 \\
\hline ZJB9-12 & 2.26 & 1.74 & 1.84 & 3.12 & 3.52 & 3.64 & 1.74 & 1.74 & 2.78 & 2.78 & 1.36 & 2.62 \\
\hline JSB1-39 & 2.46 & 1.88 & 1.86 & 3.44 & 3.88 & 3.88 & 1.72 & 1.8 & 2.8 & 2.26 & 1.3 & 2.52 \\
\hline Average $^{\mathrm{d}}$ & 1.77 & 1.26 & 2.30 & 1.46 & 2.59 & 2.72 & 2.70 & 2.21 & 1.82 & 1.74 & 1.91 & 1.97 \\
\hline
\end{tabular}

${ }^{a}$ Strains are abbreviated by the letters indicating their places isolated. AH = Anhui, GD = Guangdong, GX = Guangxi, HAN = Hainan, HN = Hunan, JS = Jiangsu, $\mathrm{SC}=$ Sichuan, $\mathrm{YN}=$ Yunnan, $\mathrm{ZJ}=\mathrm{Zhejiang}$, and $\mathrm{RS}=$ rice streak.

${ }^{\mathrm{b}}$ Bacterial leaf streak (BLS) lesion length (cm). Resistance genes against bacterial leaf blight contained in near-isogenic lines are referred from Verdier et al. (42).

c Mean of bacterial leaf streak (BLS) lesion length caused by one strain in 23 rice lines.

${ }^{\mathrm{d}}$ Mean of BLS lesion length caused by 65 strains in one rice line. 
TABLE 1. (Continued from preceding page)

\begin{tabular}{|c|c|c|c|c|c|c|c|c|c|c|c|}
\hline \multicolumn{12}{|c|}{ Lesion length $(\mathrm{cm})$ for rice lines and genes shown ${ }^{b}$} \\
\hline IRBB8 & IRBB 10 & IRBB11 & IRBB11(T) & IRBB13 & IRBB14 & IRBB21 & Java14 & Tetep & TN1 & Wase Aikoku & \\
\hline Xa8 & Xa10 & Xa11 & Xa11 & xa13 & $\mathrm{Xa14}$ & $X a 21$ & $X a 1, X a 3$ & $X a 2$ & $\mathrm{Xa14}$ & Xa3 & Average $^{\mathrm{c}}$ \\
\hline 1.22 & 0.78 & 0.88 & 0.44 & 1.3 & 0.82 & 1.36 & 0.9 & 0.98 & 0.92 & 1.48 & 1.09 \\
\hline 1.76 & 1.38 & 1.46 & 0.48 & 0.4 & 0.78 & 0.98 & 0.88 & 0.48 & 0.94 & 1.62 & 1.18 \\
\hline 3.74 & 1.9 & 2.38 & 1.86 & 1.36 & 1.46 & 1.82 & 2.26 & 1.78 & 1.24 & 2.24 & 2.01 \\
\hline 3.38 & 1.72 & 1.84 & 1.94 & 1.74 & 1.36 & 2.12 & 2.3 & 1.7 & 1.36 & 2.26 & 1.95 \\
\hline 3.7 & 1.56 & 1.56 & 1.86 & 1.44 & 1.44 & 2.24 & 2.88 & 1.62 & 1.42 & 2.14 & 1.99 \\
\hline 3.54 & 1.92 & 1.84 & 2.36 & 1.76 & 1.38 & 1.86 & 2.9 & 1.76 & 1.44 & 2.34 & 2.02 \\
\hline 3.58 & 1.78 & 2.3 & 1.78 & 1.4 & 1.34 & 1.68 & 2.84 & 1.88 & 1.46 & 2.12 & 1.96 \\
\hline 3.7 & 2 & 1.68 & 2.2 & 1.7 & 1.48 & 2.22 & 2.44 & 1.64 & 1.36 & 2.34 & 2.01 \\
\hline 3.56 & 2.22 & 1.94 & 1.74 & 1.8 & 1.8 & 2.2 & 2.62 & 1.9 & 1.26 & 2.12 & 2.06 \\
\hline 3.58 & 2.38 & 2.4 & 2.2 & 1.92 & 1.44 & 1.78 & 2.88 & 1.86 & 1.38 & 2.18 & 2.04 \\
\hline 3.56 & 1.74 & 2.46 & 1.82 & 1.4 & 1.64 & 1.72 & 2.7 & 1.84 & 1.36 & 2.06 & 1.96 \\
\hline 3.66 & 2.3 & 2.14 & 1.54 & 1.76 & 1.3 & 1.74 & 2.6 & 1.76 & 1.34 & 2.44 & 2.03 \\
\hline 3.68 & 1.72 & 2.38 & 1.8 & 1.84 & 1.4 & 2.24 & 2.74 & 1.8 & 1.48 & 1.74 & 1.99 \\
\hline 3.4 & 2.08 & 2.36 & 1.86 & 1.54 & 1.48 & 1.84 & 2.74 & 1.84 & 1.3 & 2.28 & 2.00 \\
\hline 3.36 & 1.92 & 2.2 & 2.24 & 1.76 & 1.8 & 1.76 & 2.22 & 1.84 & 1.36 & 2.32 & 2.04 \\
\hline 3.36 & 1.64 & 1.78 & 1.9 & 1.76 & 1.4 & 1.7 & 2.24 & 2.38 & 1.36 & 1.84 & 1.93 \\
\hline 3.42 & 1.8 & 2.48 & 2.22 & 2.3 & 1.84 & 1.8 & 2.74 & 2.44 & 1.34 & 2.02 & 2.19 \\
\hline 3.7 & 1.52 & 1.78 & 2.36 & 2.28 & 1.72 & 2.3 & 2.52 & 2.34 & 1.84 & 2.26 & 2.16 \\
\hline 3.4 & 2.2 & 1.62 & 1.84 & 1.8 & 1.94 & 1.82 & 2.56 & 2.42 & 1.3 & 2.06 & 2.01 \\
\hline 3.56 & 2.34 & 1.76 & 1.74 & 1.8 & 1.84 & 1.82 & 2.3 & 2.08 & 1.32 & 2.22 & 2.00 \\
\hline 3.62 & 1.8 & 2.36 & 1.82 & 1.82 & 1.88 & 1.74 & 2.78 & 2.36 & 1.46 & 2.44 & 2.06 \\
\hline 1.46 & 2.26 & 1.92 & 1.78 & 3.24 & 2.6 & 1.58 & 2.86 & 3.32 & 2.1 & 1.6 & 2.15 \\
\hline 1.42 & 1.72 & 1.82 & 2.78 & 3.32 & 2.72 & 3.24 & 2.78 & 3.44 & 2.46 & 1.88 & 2.22 \\
\hline 1.76 & 3.44 & 3.12 & 2.36 & 1.8 & 5.02 & 0.86 & 1.46 & 2.28 & 1.28 & 2.3 & 2.43 \\
\hline 2.68 & 2.48 & 4.28 & 2.14 & 3.7 & 3.32 & 3.36 & 2.84 & 2.46 & 1.88 & 2.36 & 2.47 \\
\hline 2.76 & 2.38 & 4.06 & 2.42 & 3.26 & 2.98 & 3.24 & 2.62 & 2.48 & 1.6 & 2.22 & 2.48 \\
\hline 2.6 & 2.12 & 4.44 & 2.76 & 2.66 & 3.36 & 3.16 & 2.64 & 2.34 & 1.86 & 2.24 & 2.41 \\
\hline 3.3 & 2.2 & 4.44 & 2.24 & 3.72 & 2.74 & 2.76 & 2.7 & 2.3 & 1.56 & 2.22 & 2.47 \\
\hline 2.74 & 2.26 & 4.26 & 2.28 & 3.86 & 3.3 & 3.32 & 2.64 & 2.38 & 1.96 & 2.34 & 2.46 \\
\hline 3.32 & 2.18 & 4.4 & 2.26 & 3.76 & 3.4 & 3.32 & 2.76 & 2.36 & 1.86 & 2.26 & 2.59 \\
\hline 3.24 & 2.08 & 4.22 & 2.5 & 3.52 & 3.2 & 3.4 & 2.24 & 2.26 & 1.72 & 2.5 & 2.45 \\
\hline 3.18 & 2.24 & 4.06 & 2.7 & 3.88 & 3.48 & 3.26 & 2.48 & 2.48 & 1.52 & 2.4 & 2.53 \\
\hline 2.3 & 2.34 & 3.88 & 2.28 & 3.24 & 2.86 & 3.22 & 3.18 & 1.28 & 2.3 & 1.32 & 2.51 \\
\hline 2.26 & 2.42 & 4.38 & 2.32 & 3.4 & 3.34 & 2.8 & 3.32 & 1.42 & 2.24 & 1.6 & 2.51 \\
\hline 2.32 & 2.18 & 4.46 & 2.4 & 3.16 & 3.24 & 3.18 & 3.26 & 1.42 & 2.32 & 1.44 & 2.56 \\
\hline 3.6 & 1.64 & 1.86 & 3.28 & 1.84 & 1.72 & 1.38 & 2.24 & 2.84 & 1.9 & 1.62 & 1.98 \\
\hline 3.76 & 1.54 & 2.1 & 3.28 & 1.82 & 1.92 & 1.38 & 2.22 & 2.84 & 2.34 & 1.84 & 2.04 \\
\hline 3.62 & 1.76 & 2.44 & 2.9 & 1.4 & 2.3 & 1.86 & 2.3 & 2.54 & 2.44 & 1.76 & 2.07 \\
\hline 3.64 & 2.48 & 1.84 & 2.3 & 1.78 & 1.86 & 1.84 & 2.78 & 3.26 & 2.44 & 1.54 & 2.13 \\
\hline 3.82 & 2.4 & 1.84 & 3.3 & 1.34 & 1.54 & 1.8 & 2.26 & 3.12 & 2.08 & 1.26 & 2.09 \\
\hline 3.64 & 2 & 2.24 & 3.28 & 1.8 & 2.36 & 1.3 & 2.24 & 2.26 & 2.36 & 1.48 & 2.17 \\
\hline 3.56 & 2.12 & 2.44 & 2.8 & 1.3 & 1.8 & 1.8 & 2.04 & 2.76 & 2.28 & 1.84 & 2.07 \\
\hline 3.72 & 1.96 & 2.42 & 2.18 & 1.54 & 1.78 & 1.3 & 2.38 & 2.62 & 2.36 & 1.42 & 1.99 \\
\hline 3.68 & 2.18 & 2.36 & 3.28 & 1.7 & 2.36 & 1.72 & 2.68 & 2.64 & 2.2 & 1.8 & 2.21 \\
\hline 3.38 & 2.24 & 1.98 & 3.2 & 4.42 & 1.88 & 1.38 & 2.14 & 2.44 & 2.36 & 1.34 & 2.13 \\
\hline 3.74 & 1.86 & 1.94 & 3.36 & 1.9 & 1.92 & 1.78 & 2.28 & 2.64 & 2.44 & 1.62 & 2.05 \\
\hline 3.68 & 2.24 & 1.94 & 2.82 & 1.84 & 1.64 & 1.42 & 2.4 & 2.24 & 2.62 & 1.78 & 2.07 \\
\hline 3.42 & 2.16 & 1.96 & 2.84 & 1.88 & 2.34 & 1.44 & 2.08 & 2.72 & 2.76 & 1.62 & 2.01 \\
\hline 3.76 & 2.24 & 2 & 3.44 & 1.34 & 1.58 & 1.4 & 2.48 & 2.9 & 2.68 & 1.58 & 2.04 \\
\hline 3.52 & 2.44 & 2.02 & 3.38 & 1.68 & 1.54 & 1.26 & 2.74 & 2.8 & 2.44 & 1.66 & 2.06 \\
\hline 3.58 & 1.8 & 1.74 & 1.84 & 1.8 & 1.86 & 1.78 & 2.2 & 2.82 & 2.3 & 1.8 & 2.00 \\
\hline 2.88 & 1.5 & 0.36 & 0.46 & 0.78 & 0.44 & 1.82 & 2.68 & 2.88 & 2.66 & 1.88 & 1.83 \\
\hline 2.34 & 3.66 & 1.74 & 1.78 & 1.72 & 2.72 & 1.84 & 2.26 & 2.3 & 1.3 & 1.58 & 2.17 \\
\hline 2.34 & 3.6 & 2.36 & 1.48 & 1.8 & 2.52 & 1.26 & 2.4 & 2.16 & 1.72 & 1.82 & 2.13 \\
\hline 2.4 & 3.46 & 1.96 & 1.72 & 1.32 & 2.72 & 1.76 & 2.34 & 2.38 & 1.24 & 1.96 & 2.15 \\
\hline 2.32 & 3.86 & 2.4 & 1.6 & 1.88 & 2.8 & 1.36 & 2.38 & 2.4 & 1.22 & 1.74 & 2.20 \\
\hline 1.88 & 3.22 & 1.6 & 1.68 & 1.86 & 2.28 & 1.64 & 2.34 & 2.48 & 1.46 & 1.94 & 2.09 \\
\hline 1.86 & 1.98 & 1.76 & 1.76 & 3.3 & 2.28 & 1.82 & 3.28 & 2.84 & 2.36 & 0.8 & 2.27 \\
\hline 1.84 & 1.5 & 1.5 & 1.74 & 2.82 & 2.28 & 1.78 & 2.86 & 2.88 & 2.34 & 0.58 & 2.15 \\
\hline 2.24 & 1.48 & 1.94 & 1.84 & 3.18 & 2.72 & 1.78 & 3.22 & 2.74 & 2.38 & 0.98 & 2.29 \\
\hline 1.76 & 2.4 & 1.78 & 1.34 & 3.32 & 2.86 & 2.22 & 3.36 & 2.62 & 2.76 & 0.82 & 2.28 \\
\hline 1.86 & 1.86 & 1.92 & 1.8 & 3.34 & 2.26 & 1.8 & 2.74 & 4.02 & 1.66 & 2.36 & 2.28 \\
\hline 3.8 & 1.64 & 3.72 & 1.78 & 2.74 & 1.44 & 2.24 & 2.56 & 4.38 & 0.74 & 1.96 & 2.20 \\
\hline 1.96 & 2.04 & 2.88 & 1.74 & 2.84 & 2.66 & 1.78 & 2.68 & 3.2 & 2.94 & 1.84 & 2.42 \\
\hline 1.84 & 2.2 & 2.56 & 1.7 & 2.8 & 2.7 & 1.48 & 2.6 & 3.36 & 2.6 & 1.58 & 2.40 \\
\hline 3.00 & 2.13 & 2.41 & 2.17 & 2.22 & 2.16 & 1.98 & 2.52 & 2.38 & 1.85 & 1.86 & $\ldots$ \\
\hline
\end{tabular}


JSB2-24 were recorded in GenBank as accession numbers KF447887 and KF460460, respectively.

Long PCR for detection of HMWBs in $X$. oryzae $p v$. oryzicola strains. Primers HMWBF (5'-GGAGTTGAGAGGTC
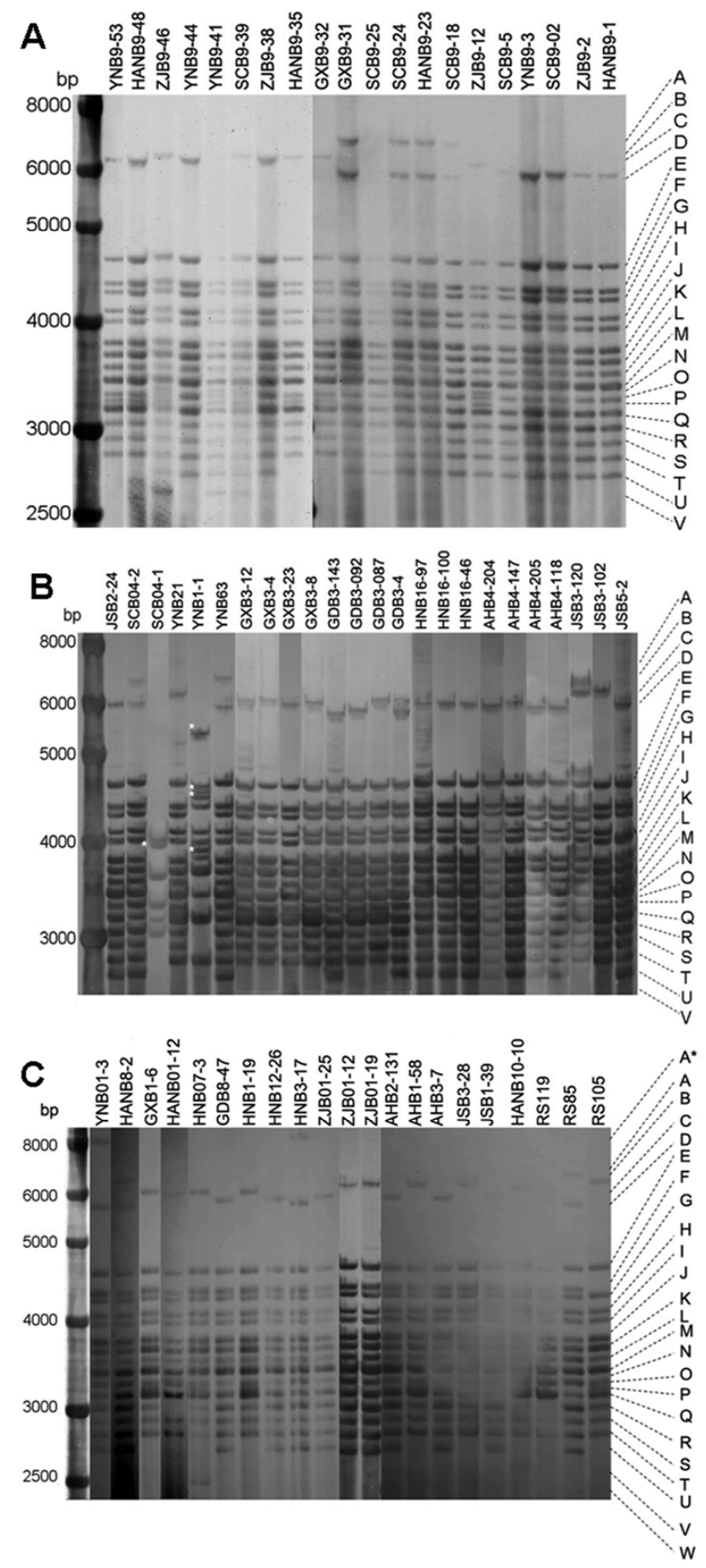

Fig. 1. Southern blot analysis of potential transcriptional activator-like effector (tale) genes in 65 strains of Xanthomonas oryzae pv. oryzicola. Genomic DNA of the strains was digested with BamHI and hybridized with an internal SphI fragment of avrXa3 (44). Molecular weight markers (bp) are shown in the left lane. Hybridizing bands of various sizes are marked with different letters $\left(\mathrm{A}^{*}, \mathrm{~A}\right.$ to $\left.\mathrm{W}\right)$, and bands marked with an asterisk were unique to a single strain. Strains were named using a letter designation by province of origin, followed by the letter B and a number. Province abbreviations are as follows: AH, Anhui; GD, Guangdong; GX, Guangxi; HAN, Hainan; HN, Hunan; JS, Jiangsu, SC, Sichuan; YN, Yunnan; and ZJ, Zhejiang. Strains designated with RS represent $X$. oryzae pv. oryzicola strains isolated from Jiangsu province prior to 2008. Southern hybridization experiments were repeated twice, with similar results.
CGCCGTTACAGTTGGACACAGGCC- $\left.3^{\prime}\right)$ and HMWBR (5'GCGGCTGCAACGCGCCGCGTGCTGCGCTGAATGGAAGC$\left.3^{\prime}\right)$ were synthesized to amplify HMWBs in representative strains of each genotype. PCR reactions were carried out in a $25-\mu 1$ reaction mixture containing $30 \mathrm{ng}$ of genomic DNA, $0.2 \mu \mathrm{M}$ each primer, $0.1 \mathrm{mM}$ each dNTP, and $2 \mathrm{U}$ of Ex-Taq DNA polymerase with GC buffer II (TaKaRa, Dalian, China). The PCR conditions were as follows: $94^{\circ} \mathrm{C}$ for $3 \mathrm{~min} ; 35$ cycles at $94^{\circ} \mathrm{C}$ for $20 \mathrm{~s}, 58^{\circ} \mathrm{C}$ for $20 \mathrm{~s}$, and $72^{\circ} \mathrm{C}$ for $3 \mathrm{~min}$; followed $72^{\circ} \mathrm{C}$ for $5 \mathrm{~min}$. PCR was performed using a Mastercycler pro $S$ (Eppendorf International). PCR products were separated by electrophoresis on $1.0 \%$ agarose gels, purified using the AxyPrep PCR clean-up kit (Axygen), and then ligated into the pMD18-T vector (TaKaRa) for sequencing (Invitrogen).

\section{RESULTS}

Isolation of $X$. oryzae pv. oryzicola strains. Diseased rice leaves showing BLS symptoms were collected from rice fields in nine provinces of southern China and processed for bacterial strain isolation. $X$. oryzae pv. oryzicola strains, showing extracellular protease activity on NA-milk plates (not all data shown) (Supplemental Figure 1), were initially identified by colony PCR using the $X$. oryzae pv. oryzicola-specific primers XoocF/XoocR (49) and $h r p E 3 F / h r p E 3 R$ (10) (not all data shown) and were then confirmed to induce HR in tobacco (data not shown) and produce BLS symptoms in the susceptible rice line IR24 (Table 1). In total, 65 pathogenic $X$. oryzae pv. oryzicola strains were isolated from the following provinces (number of strains isolated in parentheses): Anhui $(n=7)$, Guangdong $(n=6)$, Guangxi $(n=6)$, Hainan $(n=7)$, Hunan $(n=7)$, Jiangsu $(n=9)$, Sichuan $(n=8)$, Yunnan $(n=8)$, and Zhejiang $(n=7)$ (Table 1). Virulence was assessed in 23 rice lines containing different BLB $R$ genes (41), and the tested strains caused typical BLS symptoms in all the tested rice lines (Table 1). Strains SCB04-1 and YNB1-1, which were originally isolated from Sichuan and Yunnan, respectively, showed reduced virulence compared with the other 63 strains (Table 1), indicating a potential genetic variation in virulence.

$X$. oryzae pv. oryzicola strains are classified into 14 genotypes. Southern blot hybridizations were performed to determine the number and the size of tale genes in the $65 X$. oryzae pv. oryzicola strains. For these experiments, the central $S p h \mathrm{I}$ fragment of $a v r X a 3$ (44) was hybridized to BamHI-digested genomic DNAs. The majority of BamHI fragments hybridizing to the probe were 2.5 to $4.8 \mathrm{~kb}$, which indicates the presence of 9 to 33 repeat units of the 102-bp sequence that is located in the conserved, central region of tale genes. Southern blot analysis indicated that the 65 strains could be subdivided into 28 unique tale haplotypes, which were designated $\mathrm{A}^{*}$ to $\mathrm{W}$ (Fig. 1). Each strain generally contained 9 to 22 hybridizing bands of 2.5 to $8.0 \mathrm{~kb}$ in size (Table 2). The most notable, visible difference among strains was the occurrence of high molecular weight BamHI fragments (HMWBs) (Fig. 1, see bands designated A to D, near $6.0 \mathrm{~kb}$ ). Strains SCB04-1 and YBN1-1 were the most unusual in terms of hybridization patterns and exhibited several unique bands (see bands marked with asterisk; Fig. 1B; Table 2). Strain SCB04-1 contained only nine BamHI fragments with homology to the $a v r X a 3$ probe (Fig. 1B). Based on the number and size of hybridizing fragments, the tested strains were classified into 14 different genotypes designated 1 to 14 by UPGMA cluster analysis (Fig. 2A; Table 2).

Our results indicated that strains collected from Yunnan, Jiangsu, and Sichuan provinces are more diverse than those collected from other regions (Tables 2 and 3). Genotypes 3 and 10 were the most common and represented 29.23 and $24.64 \%$ of the 65 strains, respectively, while genotypes $1,2,5,7,11$, and 12 were rare and consisted of a single strain (Table 3). Strains SCB04-1 and YNB1-1, which showed unusual hybridization patterns (Fig. 1), were assigned to genotypes 1 and 2, respectively. 
Assignment of $X$. oryzae pv. oryzicola strains to 14 pathotypes. The $65 X$. oryzae pv. oryzicola strains were inoculated into 23 rice lines containing different $R$ genes for BLB and assessed for virulence. All $65 X$. oryzae pv. oryzicola strains caused typical water-soaked BLS lesions in the 23 rice lines when evaluated 6 days post inoculation, indicating that rice lines with BLB resistance are susceptible to the $X$. oryzae pv. oryzicola strains. BLS lesion lengths were 0.34 to $5.02 \mathrm{~cm}$ (Table 1). Strains SCB04-1 (containing 9 putative tale genes, genotype 1) and YNB1-1 (16 putative tale genes, genotype 2) showed attenuated virulence in rice line DV85, with mean lesion lengths of 0.42 and $0.34 \mathrm{~cm}$, respectively (Table 1). In contrast, genotype 8 , containing 22 putative tale genes (Fig. 2; Table 2), displayed hypervirulence in the rice lines, with a mean lesion length of $2.53 \mathrm{~cm}$ (Table 4). These results suggest a potential correlation between $X$. oryzae pv. oryzicola virulence and the number of putative tale genes in a particular strain. In contrast, rice lines BJ1 (containing $x a 5$ and $x a 13$ ) and DV85 (xa5, Xa7, xa13, and Xa24) $(32,41)$ were somewhat less susceptible to the $X$. oryzae pv. oryzicola strains than the other rice lines (Tables 1 and 4) and exhibited BLS mean lesion lengths of 1.27 and $1.48 \mathrm{~cm}$, respectively (Table 1). Conversely, rice lines IRBB8 (Xa8), IR26 (Xa4), IR24 (Xa18), and Java 14 (Xa1,Xa3) $(32,41)$ were more susceptible to the $X$. oryzae pv. oryzicola strains and developed mean lesion lengths of $3.03,2.74,2.60$, and $2.55 \mathrm{~cm}$, respectively (Table 1 ). These results suggest that BLS resistance and $X$. oryzae pv. oryzicola virulence is quantitative and controlled by multiple genes in the host (rice) and pathogen ( $X$. oryzae pv. oryzicola).

To determine whether the acquisition of multiple tale genes in $X$. oryzae pv. oryzicola is correlated with virulence, we sought to cluster pathotypes of the $65 X$. oryzae pv. oryzicola strains using the SIMQUAL subroutine in NTSYS (18). When a 50\% matching coefficiency was applied, the 65 strains were discriminated into 14 pathotypes (designated I to XIV) based on virulence in the 23 rice lines (Fig. 2B). Interestingly, most of the strains clustered by genotype were assigned to the same pathotype (Fig. 2), although there were several exceptions. For example, strains HANB10-10 (genotype 13) and GXB1-6 (genotype 10) clustered in pathotype III, strains RS85 (genotype 6) and GDB8-47 (genotype 3) were assigned to pathotype X, and JSB3-102 (genotype 10) was classified as pathotype VI (Fig. 2).

Analysis of HMWBs and PCR analysis. With the exception of SCB04-1 and YNB1-1, X. oryzae pv. oryzicola strains contained a large, $\approx 6.0-\mathrm{kb}$ Bam $\mathrm{HI}$ fragment that hybridized with the $a v r X a 3$ probe (Figs. 1 and $3 \mathrm{~A}$ ). Based on the common structure of mature tale genes (Fig. 3B), this $6.0-\mathrm{kb}$ Bam HI fragment (HMWB) could contain up to 35 repeats of the 102-bp conserved sequence. This prompted us to investigate the fine structure of the 6.0-kb HMWB and determine whether this locus could be used in genotyping $X$. oryzae pv. oryzicola strains by PCR. To address these objectives, we isolated the 6.3- and 6.5-kb HMWBs from strains JSB2-24 and RS105, respectively (Fig. 3A, black arrows), and cloned these fragments into pBluescript. The sequenced HMWBs were designated HMWB-a (TAL2-24) and HMWB-b (TAL105); these are depicted in Figure 3B and were deposited as GenBank accession numbers KF447887 and KF460460, respectively. Sequence analysis showed that these loci were similar in structure. Both loci contained a truncated tale lacking the C-terminal region that encodes LZ, NLS, and AD (Fig. 3B). Furthermore, both loci encoded IS1403, phosphoglycerate mutase (pgm), and endonuclease $\mathrm{V}$ (enaV). The two loci differed in the number of repeat units of the 102-bp conserved sequence; HMWB-a (tal2-24) and HMWB-b (tall05) contained 19.5 and 21.5 repeat units, respectively (Fig. 3B). Interestingly, this locus was also identified in the genome (AAQN01000001) of $X$. oryzae pv. oryzicola strain BLS256 (5); the annotated region (tal2h) encodes a C-terminally truncated tale and XOC_1555, XOC_1554 and $X O C_{-} 1553$ (Fig. 3B). The latter three genes share $98 \%$ nucleotide identity to ISI403 (24), pgm, and enaV (data not shown). In tal2 $h^{\prime}$, the number of repeat units of the 102-bp sequence is 20.5. These data suggest that the HMWB loci are conserved but vary in the number of repeat units. It should be mentioned that HMWB homologues were not detected in the genomes of $X$. oryzae pv. oryzae strains MAFF311018, KACC10331, and $\mathrm{PXO}^{\mathrm{A}}$ (BLAST analysis; data not shown). Multiple sequence alignment using mature TALEs, including AvrXa3 (accession number AY129298.1), AvrXa10 (AAA92974.1) (Fig. 3B), and AvrXa27 (AAY54168.1) revealed the absence of a 58-aa sequence at the N-terminus of TAL2-24, TAL105, and TAL2 $\mathrm{h}^{\prime}$, respectively

TABLE 2. Transcriptional activator-like effector (tale) genotypes of Xanthomonas oryzae pv. oryzicola strains collected from different rice-growing regions of south China from 2008 to 2009

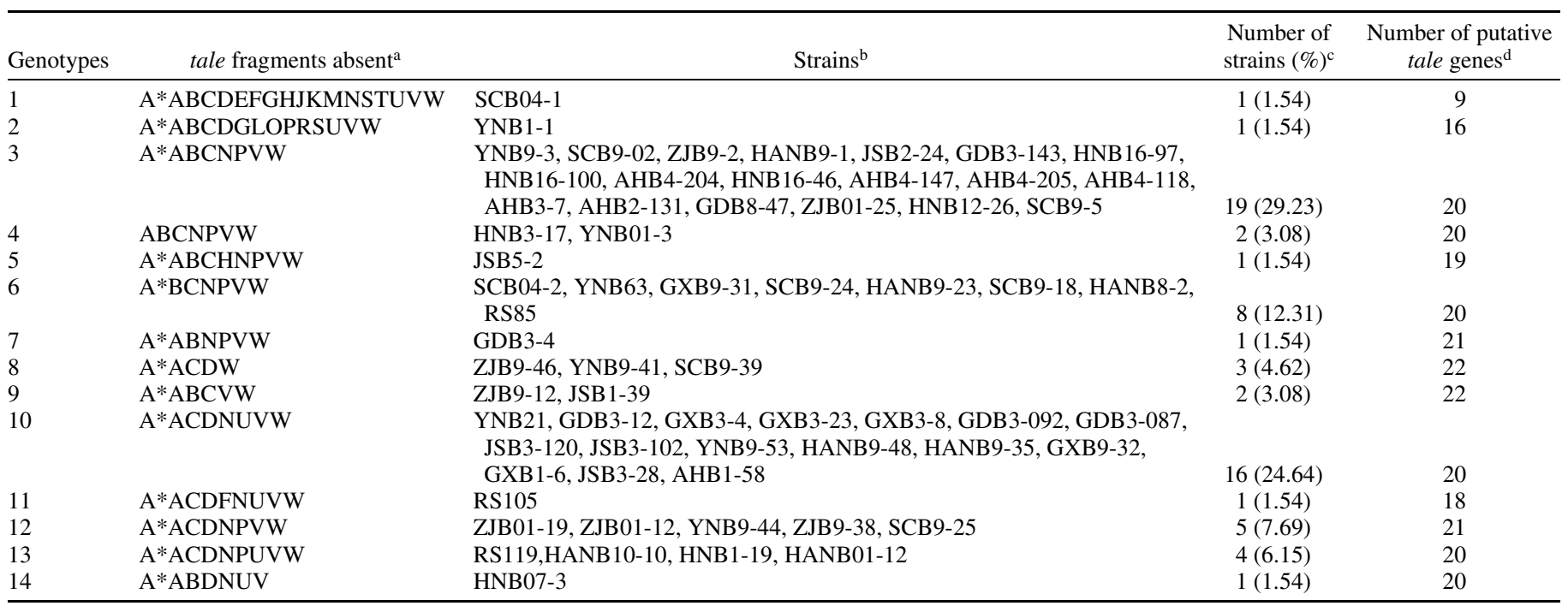

a The tale fragments absent in the tested strains. Letters $\left(\mathrm{A}^{*}, \mathrm{~A}-\mathrm{W}\right)$ represent tale fragments or bands of the tested isolates hybridized by Southern blot with the central SphI fragment of avrXa3 as the probe.

${ }^{\mathrm{b}}$ Strains are abbreviated by letters indicating their place of isolation: AH = Anhui, GD = Guangdong, GX = Guangxi, HAN = Hainan, HN = Hunan, JS = Jiangsu, $\mathrm{SC}=$ Sichuan, $\mathrm{YN}=$ Yunnan, and $\mathrm{ZJ}=$ Zhejiang; $\mathrm{RS}=$ rice streak.

${ }^{c}$ Values in parentheses indicate percentage of a genotype of the total strains.

${ }^{\mathrm{d}}$ Number of possible tale genes are estimated based on the number and size of the hybridized bands by Southern blot. 
(Supplemental Figure 2); whether this deletion is significant in pathogen virulence or host specificity awaits further investigation.

To evaluate whether the HMWBs could be used for detection of $X$. oryzae pv. oryzicola by PCR, we designed primers HMWBF and HMWBR. HMWBF maps upstream of the first repeat unit in tal2-24, tal105, and tal2 $h^{\prime}$, and primer HMWBR is located at the stop codon of IS1403 (Fig. 3B, panel b, horizontal arrows). The primers were then used in PCR with representative strains of 14 genotypes. PCR products were amplified from 12 of the genotypes, and the size of PCR products (Fig. 3C) was consistent with the calculation based on HMWBF locus structures (Fig. 3B) detected by Southern blot analysis (Fig. 3A), implying that these primers can be used to genotype $X$. oryzae pv. oryzicola strains isolated from rice-growing regions. The PCR products shown in Figure $3 \mathrm{C}$ were also sequenced, and subsequent analysis indicated that PCR product length was dependent on the number of repeat units in the C-terminally truncated portion of the HMWBs (data not shown). It is also important to note that PCR products were not detected using strains SCB04-1 and YNB1-1 (Fig. 3C), which is consistent with the absence of HMWB loci in these two strains.
A

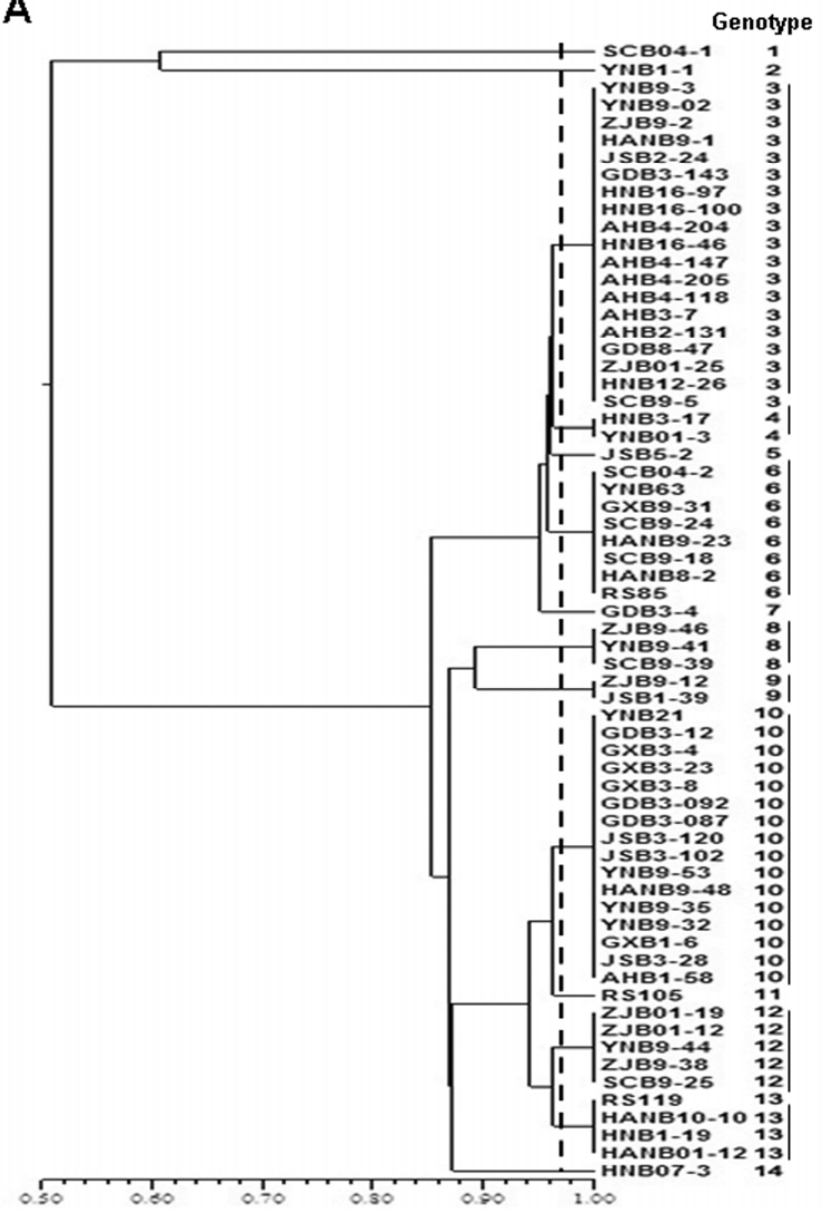

B

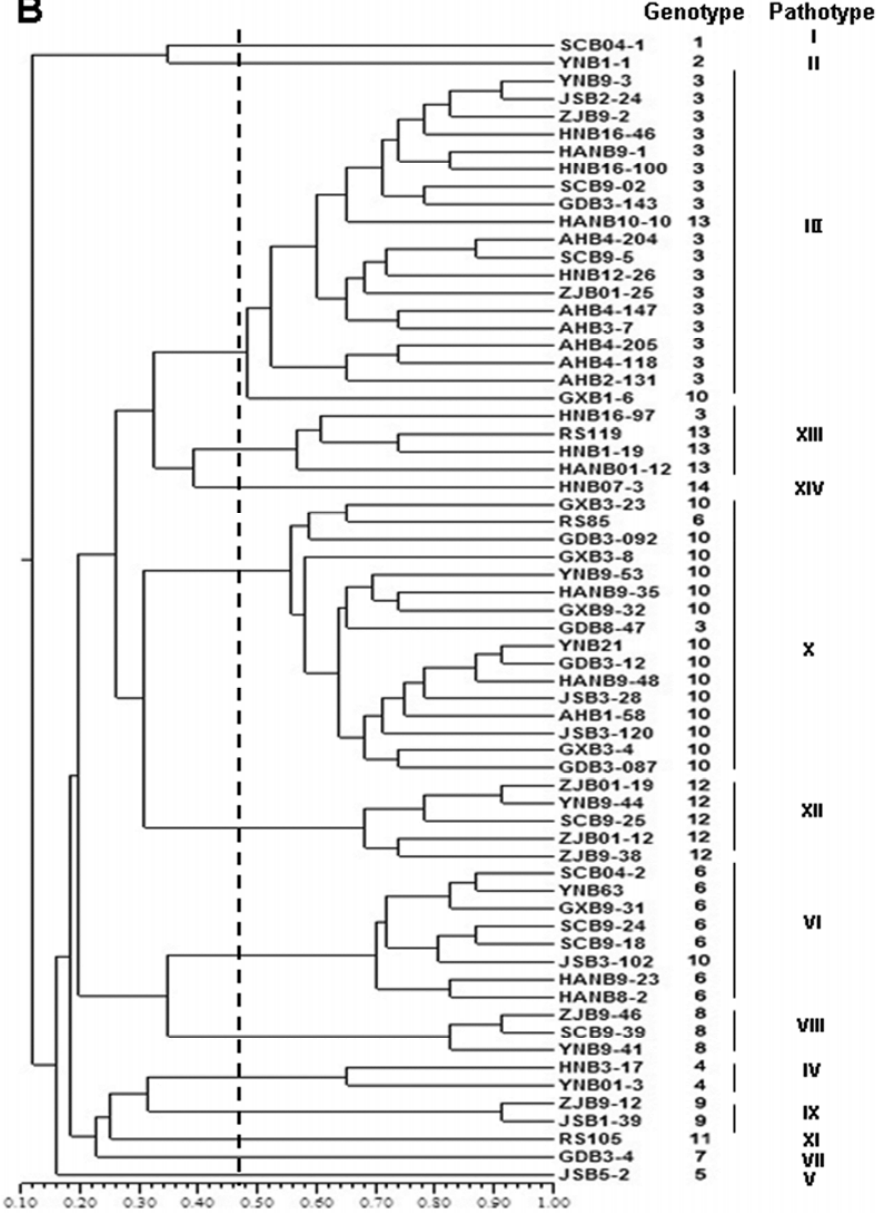

Fig. 2. Classification of 65 Xanthomonas oryzae pv. oryzicola strains into distinct genotypes and pathotypes. A, Dendrogram showing relatedness of $X$. oryzae pv. oryzicola strains based on Southern blot analysis and the presence or absence of hybridizing BamHI fragments. The dendrogram was obtained by cluster analysis using the unweighted pair group method (UPGMA) in SIMQUAL with the Dice coefficient of NTSYS $(18,25)$. Clusters were delineated at an arbitrary level of similarity equal to 0.97 . B, Dendrogram based on virulence assessment of $X$. oryzae pv. oryzicola strains inoculated to 23 different rice lines containing different resistance genes for bacterial leaf blight. The dendrogram was constructed by cluster analysis using UPGMA with the arithmetic mean method in the SAHN routine of NTSYS (18). Pathotypes were designated I to XIV and clustered at an arbitrary level of similarity equal to 0.5.

TABLE 3. Genotypes of Xanthomonas oryzae pv. oryzicola strains collected from different provinces of south China

\begin{tabular}{|c|c|c|c|c|c|c|c|c|c|c|c|c|c|c|c|}
\hline \multirow[b]{2}{*}{ Province } & \multicolumn{14}{|c|}{ Genotypes $^{\mathrm{a}}$} & \multirow[b]{2}{*}{ Strains } \\
\hline & 1 & 2 & 3 & 4 & 5 & 6 & 7 & 8 & 9 & 10 & 11 & 12 & 13 & 14 & \\
\hline Anhui & $\ldots$ & $\ldots$ & 6 & $\ldots$ & $\ldots$ & $\ldots$ & $\ldots$ & $\ldots$ & $\ldots$ & 1 & $\ldots$ & $\ldots$ & $\ldots$ & $\ldots$ & 7 \\
\hline Jiangsu & $\ldots$ & $\ldots$ & 1 & $\ldots$ & 1 & 1 & $\ldots$ & $\ldots$ & 1 & 3 & 1 & $\ldots$ & 1 & $\ldots$ & 9 \\
\hline Hunan & $\ldots$ & $\ldots$ & 4 & 1 & $\ldots$ & $\ldots$ & $\ldots$ & $\ldots$ & $\ldots$ & $\ldots$ & $\ldots$ & $\ldots$ & 1 & 1 & 7 \\
\hline Hainan & $\ldots$ & $\ldots$ & 1 & $\ldots$ & $\ldots$ & 2 & $\ldots$ & $\ldots$ & $\ldots$ & 2 & $\ldots$ & $\ldots$ & 2 & $\ldots$ & 7 \\
\hline Guangdong & $\ldots$ & $\ldots$ & 2 & $\ldots$ & $\ldots$ & $\ldots$ & 1 & $\ldots$ & $\ldots$ & 3 & $\ldots$ & $\ldots$ & $\ldots$ & $\ldots$ & 6 \\
\hline Guangxi & $\ldots$ & $\ldots$ & $\ldots$ & $\ldots$ & $\ldots$ & 1 & $\ldots$ & $\ldots$ & $\ldots$ & 5 & $\ldots$ & $\ldots$ & $\ldots$ & $\ldots$ & 6 \\
\hline Yunnan & $\ldots$ & 1 & 1 & 1 & $\ldots$ & 1 & $\ldots$ & 1 & $\ldots$ & 2 & $\ldots$ & 1 & $\ldots$ & $\ldots$ & 8 \\
\hline Sichuan & 1 & $\ldots$ & 2 & $\ldots$ & $\ldots$ & 3 & $\ldots$ & 1 & $\ldots$ & $\ldots$ & $\ldots$ & 1 & $\ldots$ & $\ldots$ & 8 \\
\hline Zhejiang & $\ldots$ & $\ldots$ & 2 & $\ldots$ & $\ldots$ & $\ldots$ & $\ldots$ & 1 & 1 & $\ldots$ & $\ldots$ & 3 & $\ldots$ & $\ldots$ & 7 \\
\hline Total & 1 & 1 & 19 & 2 & 1 & 8 & 1 & 3 & 2 & 16 & 1 & 5 & 4 & 1 & 65 \\
\hline
\end{tabular}

a Number of strains belonging to one genotype.

b Total strains collected in this province. 
TABLE 4. Virulence comparison of 14 genotypes of Xanthomonas oryzae pv. oryzicola in rice lines containing different resistance genes against bacterial leaf blight

Lesion length $(\mathrm{cm})$ for genotypes

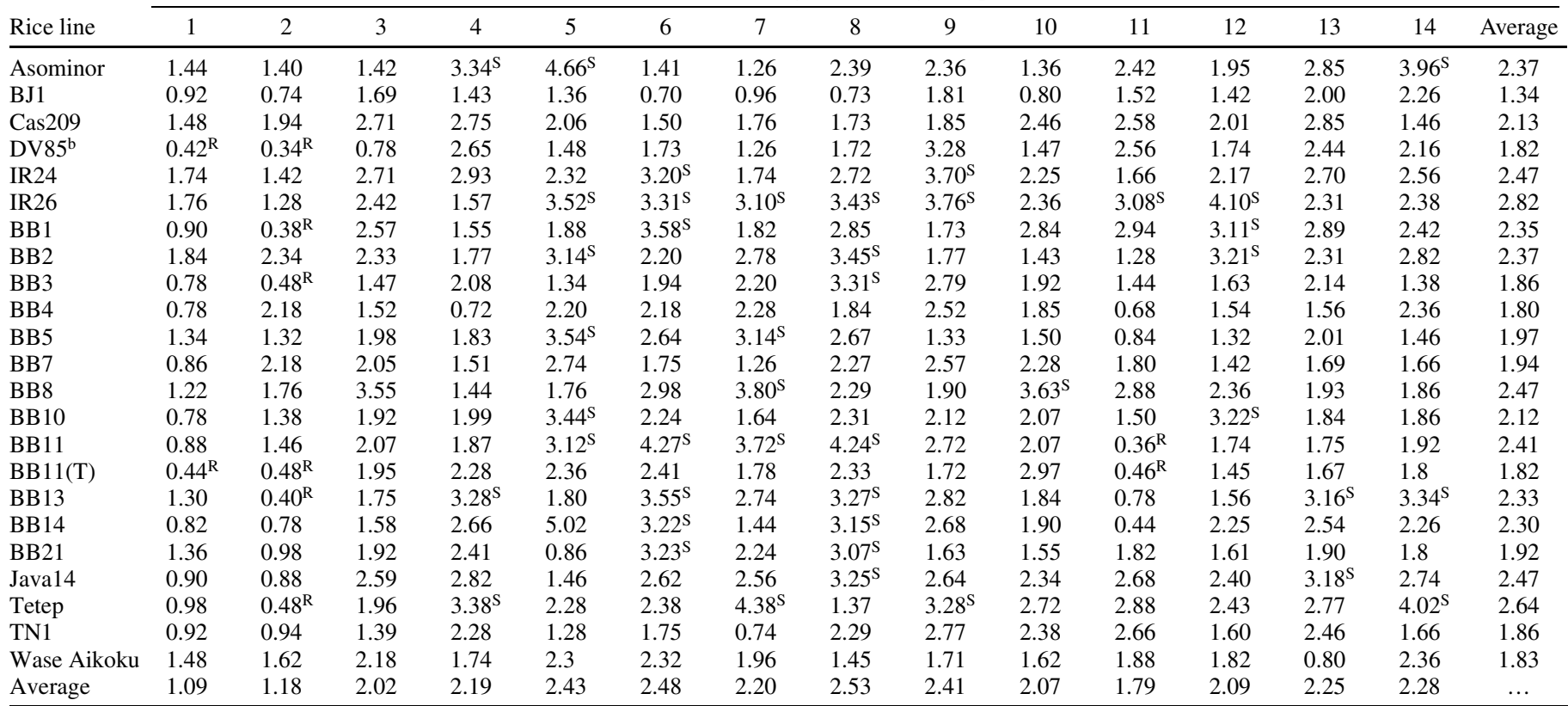

${ }^{a}$ Lesion lengths in mean caused by each genotype. $\mathrm{R}=$ resistant to the tested genotypes if lesion length is $<0.5 \mathrm{~cm}$ and $\mathrm{S}=$ more susceptible to the tested genotypes if lesion length is $>3.0 \mathrm{~cm}$.

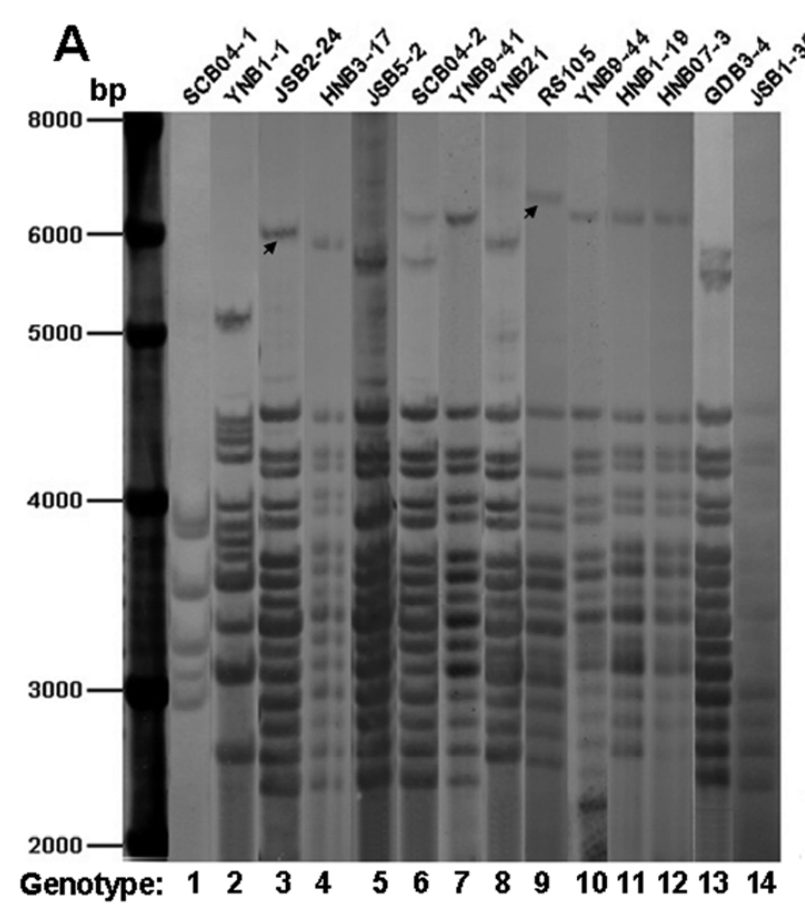

B
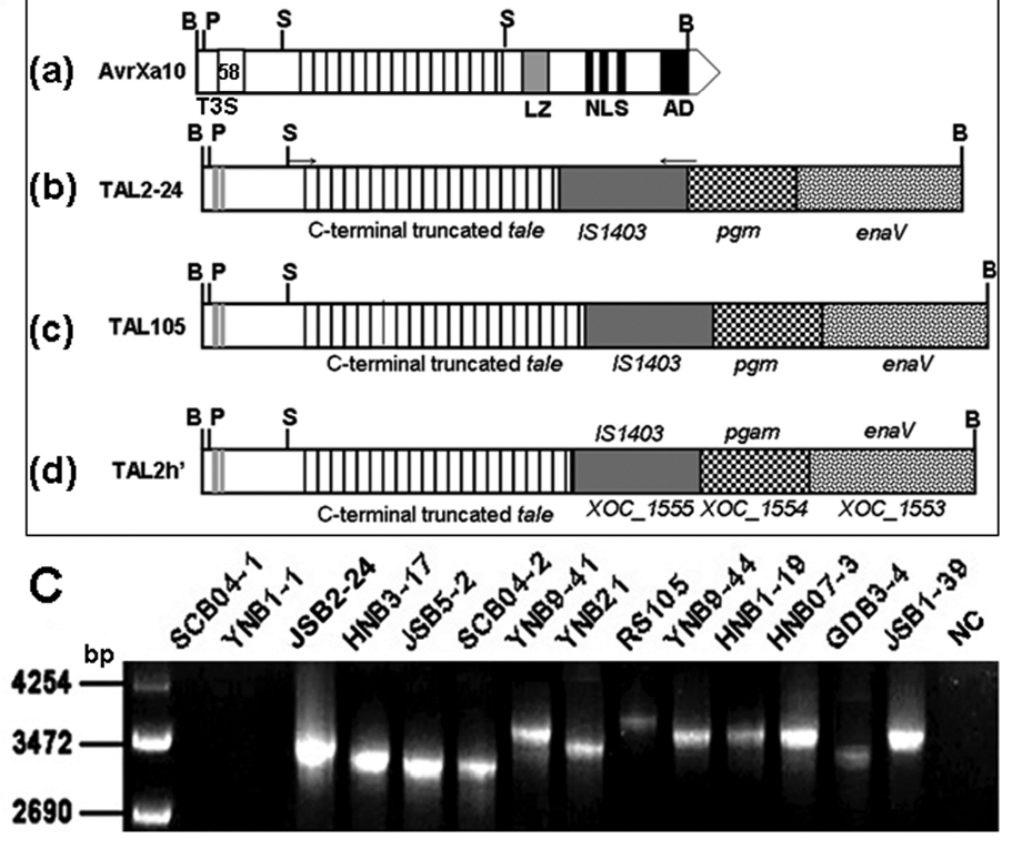

Fig. 3. Analysis of a high molecular weight band (HMWB) in the 14 genotypes of Xanthomonas oryzae pv. oryzicola. A, Southern blot analysis of strains representing the 14 genotypes of $X$. oryzae pv. oryzicola. Genomic DNAs of the strains were digested with BamHI and hybridized with an internal SphI fragment of $a v r X a 3$ (44). Bands demarcated with black arrows were isolated and sequenced. B, Fine-structure maps of a known transcriptional activator-like effector (tale) gene product and HMWBs identified in the present study. (a) Fine structure of the mature TALE encoded by avrXa10. T3S, type III secretion signals at the Nterminus of a mature TALE; LZ, leucine zipper; NLS, nuclear localization signal; AD, acidic transcriptional activation domain; B, BamHI; P, PstI; S, SphI; 58, amino acid sequence of 58 residues that is present in AvrXa10. (b) Functional map of the HMWB locus in strain JSB2-24 (designated TAL2-24). This locus is predicted to contain an immature tale with a C-terminal truncation, insertion element ISI403, phosphoglycerate mutase (pgm), and endonuclease V (enaV). The number of repeat units in TAL2-24 is 19.5. (c) Functional map of the HMWB locus in strain RS105 (designated TAL105). This locus contains all the components present in TAL2-24 but contains 21.5 repeat units in the central region. (d) Functional map of the HMWB locus in strain BLS256 (accession CP003057.1). This locus contains an immature tale (designated TAL2h'), XOC_1555, XOC_1554, and XOC_1553. The latter three genes show 98\% nucleotide identity to IS1403, pgm, and enaV in strains JSB2-24 and RS105. TAL2h' contains 20.5 repeat units. C, Detection of polymorphisms in the HMWB loci by polymerase chain reaction (PCR) amplification. Positions of the specific primers used in strain genotyping are shown by the horizontal arrows in B (b). PCR products were separated by electrophoresis in a $1 \%$ agarose gel. PCR was performed twice with similar results, and products were sequenced. NC represents that water was used as the template (negative control). Molecular size markers (bp) are indicated to the left. 


\section{DISCUSSION}

In this study, we evaluated genetic diversity within putative tale genes in $X$. oryzae pv. oryzicola strains isolated from rice-growing provinces in southern China. When an internal fragment of avrXa3 was used as a probe (44), 65 X. oryzae pv. oryzicola strains (Table 1) were clustered into 14 genotypes containing 9 to 22 potential tale genes (Table 2; Figs. 1 and 3). The majority of strains were assigned to genotypes 3 and 10 (Tables 2 and 3). There was an obvious absence of the "gene-for-gene" resistance paradigm when these 65 strains were inoculated to 23 rice lines harboring different $R$ genes for BLB $(32,41)$. The 65 strains were classified into 14 pathotypes (I to XIV) by UPGMA cluster analysis, and there was a strong correlation between pathotype and genotype groupings (Fig. 2). Furthermore, the acquisition of greater numbers of tale genes was associated with increased virulence (Tables 1, 2, and 4), supporting the contention that tale genes are involved in pathogenicity. Furthermore, we demonstrate the potential use of the HMWB loci in genotyping $X$. oryzae pv. oryzicola strains by PCR (Fig. 3C), and we propose that molecular typing using tale genes may be a more useful approach for $X$. oryzae pv. oryzicola than random amplified polymorphic DNA; AFLP; RFLP; and BOX-, ERIC-, and Rep-PCR (1,13,18,22, $34,35)$ for $X$. oryzae pv. oryzicola.

In the related pathogen, $X$. oryzae pv. oryzae, TALEs with substantial effects on virulence were shown to activate sucrose transporter genes of the OSSWEET family to promote disease development $(8,42)$. However, $X$. oryzae pv. oryzicola TALEs are not known to target the OSSWEET family in rice. It is likely that tale genes in $X$. oryzae pv. oryzicola also have specific targets in rice, because the tale gene products in other Xanthomonas spp. are known to localize to plant nuclei $(2,4,9,11,14,19,26,29,36,42)$. Thus, it is tempting to speculate that $X$. oryzae pv. oryzicola uses TALEs to promote BLS susceptibility in rice, perhaps using mechanisms that are quite different from $X$. oryzae pv. oryzae. Several pieces of evidence support this hypothesis: (i) $X$. oryzae pv. oryzicola has the ability to suppress avrXa10-Xa10-mediated resistance (27); (ii) Xa27 resistance can be triggered by X. oryzae pv. oryzicola when this pathogen harbors avrXa27 from $X$. oryzae pv. oryzae (39); (iii) BLB is a vascular disease caused by $X$. oryzae pv. oryzae whereas BLS occurs in the leaf parenchyma, implying very different infection arsenals for the two pathogens; and (iv) $X$. oryzae pv. oryzicola TALEs lack an 8-aa peptide (SGSVGGTI) that is present at the C-terminus of $X$. oryzae pv. oryzae TALEs (7). The final observation may alter the functionality of TALEs, an observation that merits further study.

It has been hypothesized that the evolution of TALEs may occur as the pathogen adapts to the selective pressure conferred by host $R$ genes $(3,30,38)$. The bacterial leaf blight pathogen, $X$. oryzae pv. oryzae, uses TALEs such as PthXo1, PthXo2, and PthXo3 $(2,46)$ to promote virulence, and it seems likely that $X$. oryzae pv. oryzicola also uses tale gene products as virulence factors. It is relevant that the $65 \mathrm{X}$. oryzae pv. oryzicola strains differed in the number of hybridizing bands (e.g., potential tale genes) and varied in virulence. For example, strain JSB5-2 containing band $\mathrm{F}$ and lacking band $\mathrm{H}$ was more virulent in rice lines Asominor and IRBB14 than strain RS105, which contains band $\mathrm{H}$ but not F (Fig. 1; Table 1). Conversely, RS105 displayed stronger virulence in rice lines DV85 and IRBB21 than JSB5-2 (Table 1). Thus, the putative tale gene encoded by band $\mathrm{F}$ may function as a virulence determinant for JSB5-2 in Asominor and IRBB14, whereas band $\mathrm{H}$ gene may be important in the virulence of RS105 in rice lines DV85 and IRBB21. Although it is tempting to correlate the presence of a particular band with virulence, such an association is highly speculative. Thus, it would be quite useful to isolate or engineer an $X$. oryzae pv. oryzicola strain that lacks TALEs and use this for reintroducing tale genes, an approach that could be used to systematically dissect the contribution of individual tale genes in virulence $(6,42)$.

An important outcome of the present study was the identification of the HMWB loci in 63 of the $65 X$. oryzae pv. oryzicola strains. Sequence analysis indicated that the HMWB loci contained a C-terminally truncated tale, and encoded IS1403 (XOC_1555), pgm (XOC_1554), and enaV (XOC_1553) (Fig. $3 \mathrm{~B})$; the latter three loci are conspicuously absent from the genomes of $X$. oryzae pv. oryzae strains KACC10331 (23), MAFF311018 (31), and PXO99A (37). It is also noteworthy that the C-terminally truncated TALEs lack a 58-aa peptide that generally maps at the $\mathrm{N}$ terminus of mature TALEs. This raises an interesting question: did the mature tale genes in $X$. oryzae $\mathrm{pv}$. oryzae strains such as AvrXa3, AvrXa10, and AvrXa27 perhaps evolve from the HMWB loci described in this study? Furthermore, some of the virulence properties might be present in the HMWB loci, which possibly represent immature, ancestral tale genes. In support of this speculation, we previously isolated an immature tale from an $X$. oryzae pv. oryzae strain which reduced $X$. oryzae pv. oryzae virulence in rice (20), suggesting that Cterminus-truncated tale genes still play roles in bacterial virulence in rice. In preliminary studies, we discovered that derivatives of tal $3 a$ and tal $3 b$ from $X$. oryzae pv. oryzae containing truncations in the C-terminus end still function in virulence (unpublished data). These truncated derivatives, like the HMWB loci described above, lack the 58-aa peptide and the NLS and AD domains. Although the mechanistic basis is unclear, these "immature" tale gene products may be transported via the T3SS into the host cytoplasm, or perhaps they bind a mature TALE for localization to the host nucleus. More detailed studies of tale gene function in $X$. oryzae pv. oryzicola will help elucidate the role of these factors in modulating the outcome of the host-pathogen interaction.

\section{ACKNOWLEDGMENTS}

This work was supported by the State Key Basic Research and Development Project of China (20112CB114003), the Natural Science Foundation of China (31230059), and the Special Fund for Agro-Scientific Research in the Public Interest of China (201303015). Z.-Y. Ji and M. Zakria contributed equally to this work. Z.-Y. Ji performed experiments; G.-Y. Chen and L.-F. Zou designed experiments; G.-H. Ji collected strains; and Z.-Y. Ji, G.-Y. Chen, and M. Zakria wrote the article.

\section{LITERATURE CITED}

1. Adhikari, T. B., Cruz , C., Zhang, Q., Nelson, R. J., Skinner, D. Z., Mew, T. W., and Leach, J. E. 1995. Genetic diversity of Xanthomonas oryzae pv. oryzae in Asia. Appl. Environ. Microbiol. 61:966-971.

2. Antony, G., Zhou, J., Huang, S., Li, T., Liu, B., White, F., Yang, B. 2010. Rice $x a 13$ recessive resistance to bacterial blight is defeated by induction of the disease susceptibility gene Os11N3. Plant Cell 22:3864-3876.

3. Boch, J., and Bonas, U. 2010. Xanthomonas AvrBs3 family-type III effectors: discovery and function. Annu. Rev Phytopathol. 48:419-436.

4. Boch, J., Scholze, H., Schornack, S., Landgraf, A., Hahn, S., Kay, S., Lahaye, T., Nickstadt, A., and Bonas, U. 2009. Breaking the code of DNA binding specificity of TAL-type III effectors. Science 326:1509-1512.

5. Bogdanove, A. J., Koebnik, R., Lu, H., Furutani, A., Angiuoli, S. V., Patil, P. B., Van Sluys, M. A., Ryan, R. P., Meyer, D. F., Han, S. W., Aparna, G., Rajaram, M., Delcher, A. L., Phillippy, A. M., Puiu, D., Schatz, M. C., Shumway, M., Sommer, D. D., Trapnell, C., Benahmed, F., Dimitrov, G., Madupu, R., Radune, D., Sullivan, S., Jha, G., Ishihara, H., Lee, S. W., Pandey, A., Sharma, V., Sriariyanun, M., Szurek, B., Vera-Cruz, C. M., Dorman, K. S., Ronald, P. C., Verdier, V., Dow, J. M., Sonti, R. V., Tsuge, S., Brendel, V. P., Rabinowicz, P. D., Leach, J. E., White, F. F., and Salzberg, S. L. 2011. Two new complete genome sequences offer insight into host and tissue specificity of plant pathogenic Xanthomonas spp. J. Bacteriol. 193:5450-5464.

6. Cao, Y., Zou, L., Zhao, W., Ji, Z., Zou, H., and Chen, G., 2011. Establishment of knockout mutagenesis in avrBs3/pthA family genes of Xanthomonas oryzae pv. oryzicola. J. Zhejiang Univ. Agric. Life Sci. 37:40-48.

7. Chen, G. Y., Zou, L. F., Wu, X. M., Li, Y. R., and Wang, J. S. 2005. 
avr/pth13 gene of Xanthomonas oryzae pv. oryzicola, a novel virulence member of avrBs3/PthA family, strengthening virulence of Xanthomonas oryzae pv. oryzae on rice. Chin. J. Rice Sci. 19:291-296.

8. Chen, L. Q., Hou, B. H., Lalonde, Takanaga, H., Hartung, M. L., Qu, X. Q., Guo, W. J., Kim, J. G., Underwood, W., Chaudhuri, B., Chermak, D., Antony, G., White, F. F., Somerville, S. C., Mudgett, M. B., and Frommer, W. B. 2010. Sugar transporters for intercellular exchange and nutrition of pathogens. Nature 468:527-532.

9. Chu, Z., Yuan, M., Yao, J., Ge, X., Yuan, B., Xu, C., Li, X., Fu, B., Li, Z., Bennetzen, J. L., Zhang, Q., and Wang, S. 2006. Promoter mutations of an essential gene for pollen development result in disease resistance in rice. Genes Dev. 20:1250-1255.

10. Cui, Y. P., Zou, L. F., Zou, H. S., Li, Y. R., Zakria, M., and Chen, G. Y. 2013. HrpE3 is a type III effector protein required for full virulence of Xanthomonas oryzae pv. oryzicola in rice. Mol. Plant Pathol. 14:678-692.

11. Deng, D., Yan, C. Y., Pan, X. J., Mahfouz, M., Wang, J. W., Zhu, J. K., Shi, Y. G., and Yan, N. 2012. Structural basis for sequence-specific recognition of DNA by TAL effectors. Science 335:720-723.

12. Fang, C. T., Ren, H. C., Chen, T. Y., Zhu, Y. K., Fan, H. C., and Wu, S. C. 1957. A comparison of the rice bacterial leaf blight organism with the bacterial leaf streak organisms of rice and Leersia hexandra Swartz. Acta Phytopathol. Sin. 3:99-124.

13. Gonzalez, C., Szurek, B., Manceau, C., Mathieu, T., Sere, Y., and Verdier, V. 2007. Molecular and pathotypic characterization of new Xanthomonas oryzae strains from West Africa. Mol. Plant-Microbe Interact. 20:534546.

14. Gu, K., Yang, B., Tian, D., Wu, L., Wang, D., Sreekala, C., Yang, F., Chu, Z., Wang, G. L., White, F. F., and Yin, Z. 2005. $R$ gene expression induced by a type-III effector triggers disease resistance in rice. Nature 435:11221125 .

15. Hajri, A., Brin, C., Zhao, S., David, P., Feng, J. X., Koebnik, R., Szurek, B., Verdier, V., Boureau, T., and Poussier, S. 2012. Multilocus sequence analysis and type III effector repertoire mining provide new insights into the evolutionary history and virulence of Xanthomonas oryzae. Mol. Plant Pathol. 13:288-302.

16. Han, Q. D., Chen, Z. W., Deng, Y., Lan, T., Guan, H. Z., Duan, Y. L., Zhou, Y. C., Lin, M. C., and Wu, W. R. 2008. Fine mapping of qBlsr5a, a QTL controlling resistance to bacterial leaf streak in rice. Acta Agron. Sin. 34:587-590.

17. He, W. A., Huang, D. H., Cen, Z. L., Zhang, Y. X., Ma, Z. F., Liu, C., Chen, Y. Z., Lu, S. N., Liu, K. Y., and Li, R. B. 2010. Research progress on rice resistance to bacterial leaf streak. J. Plant Genet. Resour. 11:116119.

18. Hu, J., Zhang, Y., Qian, W., and He, C. 2007. Avirulence gene and insertion element-based RFLP as well as RAPD markers reveal high levels of genomic polymorphism in the rice pathogen Xanthomonas oryzae pv. oryzae. Syst. Appl. Microbiol. 30:587-600.

19. Hummel, A. W., Doyle, E. L., and Bogdanove, A. J. 2012. Addition of TAL effector binding sites to a pathogen strain-specific rice bacterial blight resistance gene makes it effective against additional strains and against bacterial leaf streak. New Phytol. 195:883-893.

20. Ji, Z. Y., Yang, J., Wang, Y. P., Zhou, D., Li, Y. R., Zou, L. F., and Chen, G. Y. 2009. Identification of a specific avirulence gene from a Chinese race C8 of Xanthomonas oryzae pv. oryzae. Chin. J. Rice Sci. 23:463-469.

21. Kay, S., Hahn, S., Marois, E., Hause, G., and Bonas, U. 2007. A bacterial effector acts as a plant transcription factor and induces a cell size regulator. Science 318:648-651.

22. Leach, J. E., Rhoads, M. L., Vera Cruz, C. M., White, F. F., Mew, T. W., and Leung, H. 1992. Assessment of genetic diversity and population structure of Xanthomonas oryzae pv. oryzae with a repetitive DNA element. Appl. Environ. Microbiol. 58:2188-2195.

23. Lee, B. M., Park, Y. J., Park, D. S., Kang, H. W., Kim, J. G., Song, E. S., Park, I. C., Yoon, U. H., Hahn, J. H., Koo, B. S., Lee, G. B., Kim, H., Park, H. S., Yoon, K. O., Kim, J. H., Jung, C. H., Koh, N. H., Seo, J. S., and Go, S. J. 2005. The genome sequence of Xanthomonas oryzae pathovar oryzae KACC10331, the bacterial blight pathogen of rice. Nucleic Acids Res. 33:577-586.

24. Lee, Y. A., and Chiu, S. P. 1998. IS1403 and IS1404: analysis and distribution of two new insertion sequences in Xanthomonas campestris. Bot. Bull. Acad. Sin. 39:231-239.

25. Lewontin, R. C. 1972. The apportionment of human diversity. Evol. Biol. 6:381-398

26. Mak, A. N., Bradley, P., Cernadas, R. A., Bogdanove, A. J., Stoddard, B. L. 2012. The crystal structure of TAL effector PthXo1 bound to its DNA target. Science 335:716-719.

27. Makino, S., Sugio, A., White, F. F., and Bogdanove, A. J. 2006. Inhibition of resistance gene-mediated defense in rice by Xanthomonas oryzae pv. oryzicola. Mol. Plant-Microbe Interact. 19:240-249.

28. Mew, T. W., Alvarez, A. M., Leach, J. E., and Swings, J. 1993. Focus on bacterial blight of rice. Plant Dis. 77:5-12.
29. Moscou, M. J., and Bogdanove A. J. 2009. A simple cipher governs DNA recognition by TAL effectors. Science 326:1501.

30. Niño-Liu, D. O., Ronald, P. C., and Bogdanove, A. J. 2006. Xanthomonas oryzae pathovars: model pathogens of a model crop. Mol. Plant Pathol. 7:303-324.

31. Ochiai, H., Inoue, Y., Takeya, M., Sasaki, A., and Kaku, H. 2005. Genome sequence of Xanthomonas oryzae pv. oryzae suggests contribution of large numbers of effector genes and insertion sequences to its race diversity. Jpn. Agric. Res. Q. 39:275-287.

32. Ogawa, T., 1993. Methods and strategy for monitoring race distribution and identification of resistance genes to bacterial leaf blight (Xanthomonas campestris pv. oryzae) in rice. Jpn. Agric. Res. Q. 27:71-80.

33. Ou, S. H. 1984. Exploring tropical rice diseases: a reminiscence. Annu. Rev. Phytopathol. 22:1-11.

34. Rademaker, J. L., Hoste, B., Louws, F. J., Kersters, K., Swings, J., Vauterin, L., Vauterin, P., and de Bruijn, F. J. 2000. Comparison of AFLP and rep-PCR genomic fingerprinting with DNA-DNA homology studies: Xanthomonas as a model system. Int. J. Syst. Evol. Microbiol. 2:665-677.

35. Raymundo, A. K., Briones, A. M., Jr., Ardales, E. Y., Perez, M. T., Fernandez, L. C., Leach, J. F., Mew, T. W., Ynalvez, M. A., McLaren, C. G., and Nelson, R. J. 1999. Analysis of DNA polymorphism and virulence in Philippine strains of Xanthomonas oryzae pv. oryzicola. Plant Dis. 83:434-440.

36. Romer, P., Recht, S., Strau $\beta$, T., Elsaesser, J., Schornack, S., Boch, J., Wang, S., Lahaye, T., 2010. Promoter elements of rice susceptibility genes are bound and activated by specific TAL effectors from the bacterial blight pathogen, Xanthomonas oryzae pv. oryzae. New Phytol. 187:1048-1057.

37. Salzberg, S. L., Sommer, D. D., Schatz, M. C., Phillippy, A. M., Rabinowicz, P. D., Tsuge, S., Furutani, A., Ochiai, H., Delcher, A. L., Kelley, D., Madupu, R., Puiu, D., Radune, D., Shumway, M., Trapnell, C., Aparna, G., Jha, G., Pandey, A., Patil, P. B., Ishihara, H., Meyer, D. F., Szurek, B., Verdier, V., Koebnik, R., Dow, J. M., Ryan, R. P., Hirata, H., Tsuyumu, S., Won Lee, S., Seo, Y. S., Sriariyanum, M., Ronald, P. C., Sonti, R. V., Van Sluys, M. A., Leach, J. E., White, F. F., and Bogdanove, A. J. 2008. Genome sequence and rapid evolution of the rice pathogen Xanthomonas oryzae pv. oryzae PXO99A. BMC Genomics 9:204.

38. Tang, D., Wu, W., Li, W., Lu, H., and Worland, A. J. 2000. Mapping of QTLs conferring resistance to bacterial leaf streak in rice. Theor. Appl. Genet. 101:286-291.

39. Tian, D., and Yin, Z., 2009. Constitutive heterologous expression of avrXa27 in rice containing the $R$ gene $X a 27$ confers enhanced resistance to compatible Xanthomonas oryzae strains. Mol. Plant Pathol. 10:29-39.

40. Triplett, L. R., Hamilton, J. P., Buell, C. R., Tisserat, N. A., Verdier, V., Zink, F., and Leach, J. E. 2011. Genomic analysis of Xanthomonas oryzae isolates from rice grown in the United States reveals substantial divergence from known $X$. oryzae pathovars. Appl. Environ. Microbiol. 77:3930-3937.

41. Verdier, V., Cruz, C. V., and Leach, J. E. 2012. Controlling rice bacterial blight in Africa: needs and prospects. J. Biotechnol. 159:320-328.

42. Verdier, V., Triplett, L. R., Hummel, A. W., Corral, R., Cernadas, R. A., Schmidt, C. L., Bogdanove, A. J., and Leach, J. E. 2012. Transcription activator-like (TAL) effectors targeting OsSWEET genes enhance virulence on diverse rice (Oryza sativa) varieties when expressed individually in a TAL effector-deficient strain of Xanthomonas oryzae. New Phytol. 196:1197-1207.

43. White, F. F., and Yang, B. 2009. Host and pathogen factors controlling the rice-Xanthomonas oryzae interaction. Plant Physiol. 150:1677-1686.

44. Wu, X. M., Li, Y. R., Zou, L. F., and Chen, G. Y. 2007. Gene-for-gene relationships between rice and diverse avrBs3/pthA avirulence genes in Xanthomonas oryzae pv. oryzae. Plant Pathol. 56:26-34.

45. Yang, B., Sugio, A., and White, F. F. 2005. Avoidance of host recognition by alterations in the repetitive and C-terminal regions of AvrXa7, a type III effector of Xanthomonas oryzae pv. oryzae. Mol. Plant-Microbe Interact. 18:142-149.

46. Yang, B., Sugio, A., and White, F. F. 2006. Os $8 N 3$ is a host diseasesusceptibility gene for bacterial blight of rice. Proc. Natl. Acad. Sci. USA 103:10503-10508.

47. Yang, B., and White, F. F. 2004. Diverse members of the AvrBs3/PthA family of type III effectors are major virulence determinants in bacterial blight disease of rice. Mol. Plant-Microbe Interact. 17:1192-1200.

48. Ye, G., Hong, N., Zou, L., Zou, H., Zakria, M., Wang, G., and Chen, G. 2013. tale-based genetic diversity of Chinese isolates of the citrus canker pathogen Xanthomonas citri subsp. citri. Plant Dis. doi:10.1094/pdis-1212-1201-RE

49. Zhang, H., Jiang, Y. H., Hu, B. S., Liu, F. Q., and Xu, Z. G. 2008. Specific detection of Xanthomonas oryzae pv. oryzicola by PCR techniques. Acta Phytopathol. Sin. 38:1-5.

50. Zhao, B., Lin, X., Poland, J., Trick, H., Leach, J., and Hulbert, S. 2005. A maize resistance gene functions against bacterial streak disease in rice. 
Proc. Natl. Acad. Sci. USA 102:15383-15388.

51. Zou, H. S., Yuan, L., Guo, W., Li, Y. R., Che, Y. Z., Zou, L. F., and Chen, G. Y. 2011. Construction of a Tn5-tagged mutant library of Xanthomonas oryzae pv. oryzicola as an invaluable resource for functional genomics. Curr. Microbiol. 62:908-916.
52. Zou, L. F., Wang, X. P., Xiang, Y., Zhang, B., Li, Y. R., Xiao, Y. L., Wang, J. S. Walmsley, A. R., and Chen, G. Y. 2006. Elucidation of the hrp clusters of Xanthomonas oryzae pv. oryzicola that control the hypersensitive response in nonhost tobacco and pathogenicity in susceptible host rice. Appl. Environ. Microbiol. 72:6212-6224. 